\title{
Measuring nanoscale diffusion dynamics in cellular membranes with super-resolution STED-FCS
}

Erdinc Sezgin ${ }^{1, *}$, Falk Schneider ${ }^{1}$, Silvia Galiani ${ }^{1}$, Iztok Urbančič ${ }^{1,6}$, Dominic Waithe ${ }^{2,3}$, B. Christoffer Lagerholm ${ }^{2}$, Christian Eggeling ${ }^{1,2,4,5, *}$

${ }^{1} \mathrm{MRC}$ Human Immunology Unit, ${ }^{2}$ Wolfson Imaging Centre, and ${ }^{3} \mathrm{MRC}$ Centre for Computational Biology, MRC Weatherall Institute of Molecular Medicine, University of Oxford, Oxford OX3 9DS, United Kingdom

${ }^{4}$ Institute of Applied Optics, Friedrich-Schiller-University Jena, Max-Wien Platz 1, 07743 Jena, Germany

${ }^{5}$ Leibniz Institute of Photonic Technology e.V., Albert-Einstein-Straße 9, 07745 Jena, Germany

6“Jožef Stefan” Institute, SI-1000, Ljubljana, Slovenia

falk.schneider@rdm.ox.ac.uk

silvia.galiani@rdm.ox.ac.uk

iztok.urbancic@,rdm.ox.ac.uk

dominic.waithe@imm.ox.ac.uk

christoffer.lagerholm@,ndcls.ox.ac.uk

Correspondence:

erdinc.sezgin@rdm.ox.ac.uk

christian.eggeling@uni-jena.de

Phone: +44-(0)1865-222-484, Fax: +44 (0) 1865222502 
KEYWORDS super-resolution microscopy, stimulated emission depletion, STED, STED microscopy, fluorescence correlation spectroscopy, FCS, STED-FCS, diffusion, plasma membrane, nanodomains, molecular interactions

EDITORIAL SUMMARY This Protocol describes how to measure nanoscale diffusion dynamics of proteins and lipids in cell membranes using STED-FCS. The protocol contains procedures for calibration, performing point- and scanning-STED-FCS measurements, and data analysis.

TWEET Measuring nanoscale diffusion dynamics in cell membranes using super-resolution STED-FCS. \#superresolution

COVER TEASER Nanoscale diffusion dynamics using STED-FCS

Up to three primary research articles where the protocol has been used and/or developed:

1. Eggeling, C. et al. Direct observation of the nanoscale dynamics of membrane lipids in a living cell. Nature 457, 1159-1162, doi:10.1038/nature07596 (2009).

2. Honigmann, A. et al. Scanning STED-FCS reveals spatiotemporal heterogeneity of lipid interaction in the plasma membrane of living cells. Nature Communications 5, 5412-5412, doi:10.1038/ncomms6412 (2014).

3. Schneider, F. et al. Diffusion of lipids and GPI-anchored proteins in actin-free plasma membrane vesicles measured by STED-FCS. Molecular Biology of the Cell 28, 1507-1518, doi:10.1101/076109 (2017). 


\begin{abstract}
Super-resolution microscopy techniques enable optical imaging in live cells with unprecedented spatial resolution. They unfortunately lack the temporal resolution required to directly investigate the cellular dynamics at scales sufficient to measure molecular diffusion. These fast time scales are, on the other hand, routinely accessible by spectroscopic techniques such as fluorescence correlation spectroscopy (FCS). To enable the direct investigation of fast dynamics at the relevant spatial scales, FCS has been combined with super-resolution stimulated emission depletion (STED) microscopy. STED-FCS has been applied in point or scanning mode to reveal nanoscale diffusion behaviour of molecules in live cells. In this protocol, we describe the technical details needed to perform point STED-FCS and scanning STED-FCS measurements, from calibration, sample preparation to data acquisition and analysis. We give particular emphasis to twodimensional diffusion dynamics in cellular membranes using molecules tagged with organic fluorophores. These measurements can be accomplished within 4-6 h by those proficient with fluorescence imaging.
\end{abstract}




\section{INTRODUCTION}

\section{Spatiotemporal scales of cellular processes and experimental methods}

The dynamic processes that govern the cellular physiology happen on different spatial and temporal scales, ranging from micrometres to nanometres and minutes to microseconds, respectively ${ }^{1}$. Cells, typically a few tens of micrometres across, are sub-compartmentalised with organelles that can be smaller than a fraction of a micrometre, which are further composed of nanometre-size molecular complexes. Cell movements and re-arrangements of organelles can take minutes, while molecular diffusion at the relevant distances occurs in the microsecond regime.

The broad range of spatiotemporal scales poses a major challenge to experimental methodologies aiming to capture the complexity of structures and dynamics in living cells. For live cell studies at the molecular level, super-resolution imaging methods, such as stimulated emission depletion (STED) microscopy, provide so far unprecedented structural details ${ }^{2}$. However, these techniques usually produce meaningful data only for structures moving slower than the required acquisition time. Therefore, these imaging techniques fail to report the dynamics of fast-moving small molecules, which is key to decipher their interactions and thereby functions. The required temporal scale can be accessed by spectroscopic methods such as fluorescence correlation spectroscopy $(\mathrm{FCS})^{3}$, but these methods are typically performed on diffraction-limited optical setups, such that any information on the nanoscale behaviour cannot be discerned. However, accurate characterisation of molecular processes in living cells requires observation techniques with the highest possible resolution in both space and time. This has been achieved by the recently developed combination of super-resolution fluorescence microscopy and spectroscopy techniques ${ }^{2,4}$, such as STED-FCS. In this Protocol we provide a step-by-step description of sample preparation, calibration, and data acquisition and analysis for STED-FCS of fluorescent lipids and proteins in live cells.

\section{Spatial resolution and stimulated emission depletion (STED) microscopy}

The spatial resolution of an optical system is its ability to distinguish nearby sample features, and is defined as the minimum distance between two objects that can be visualised as two distinct entities. For conventional far-field optical microscopy (i.e. microscopy employing lenses to focus 
the light on the sample), the resolution is limited by the diffraction of light to approximately half the wavelength of the light used, and according to Abbe this resolution limit (d) (also referred to as the diffraction limit) is determined by:

$$
d=0.5 \frac{\lambda}{N A_{o b j}}(\text { Eq. 1) }
$$

where $\lambda$ is the wavelength of the light and $N A_{o b j}$ is the numerical aperture of the imaging system (i.e., the refractive index of the medium between the objective lens and the sample, multiplied by the sine of the angle at which the objective lens focuses or collects the light). Using modern liquidimmersion objectives, NAs of up to 1.45 can be achieved and this can go up to 1.7 with very special equipment. With an oil immersion objective with NA of 1.40 , this yields a resolution for visible light at $\lambda=488 \mathrm{~nm}$ of $\mathrm{d} \approx 170 \mathrm{~nm}$ and for $\lambda=640 \mathrm{~nm}$ of $\mathrm{d} \approx 230 \mathrm{~nm}$.

The diffraction limit, as a phenomenon, cannot be simply overcome. However, the specific features of fluorescence emission can be exploited to circumvent this limit, as has been done with superresolution microscopy approaches ${ }^{2}$. For example, STED microscopy, which was formulated in 90s and experimentally realized in later years ${ }^{5-7}$, is based on reversibly driving a fluorophore between an on- and off-state ${ }^{7}$. The off-switching of the detected fluorescence signal is realised through the de-excitation of fluorescent molecules by stimulated emission (depletion) using an additional STED laser that features a local intensity zero (for example a doughnut-shaped intensity distribution in the xy-plane). This results in shrinking the effective observation volume to its very centre by selective depletion of all excited fluorophores at the periphery of the local zero (Figure 1a). The lateral size of the non-depleted region and thus the effective observation volume (diameter $d_{S T E D)}$ gradually decreases with increasing intensity of the STED laser:

$$
d_{S T E D}=\frac{d}{\sqrt{1+A^{I} \frac{I_{T E D}}{I_{S}}}} \text { (Eq. 2) }
$$

Where $d$ is the diameter of the diffraction-limited excitation laser spot, A $(>0)$ is a geometrical parameter that takes the shape of the STED laser beam into account, ISTED is the STED laser intensity and $I_{S}$ is the saturation intensity. $I_{S}$ is a characteristic property of a specific fluorescence label that embodies its depletion efficiency by the STED laser. In theory, the spatial resolution can 
be pushed to unlimited scales, but in practice lateral resolution of $\approx 20-50 \mathrm{~nm}$ has been achieved for cell studies ${ }^{2}$ due to the photophysical limitations of the fluorophores under physiological conditions.

\section{Temporal resolution and fluorescence correlation spectroscopy (FCS)}

STED microscopy imaging, like confocal microscopy, is based on sequential raster-scanning of the sample, which renders image acquisition too slow to track individual diffusing molecules. To characterise fast diffusion dynamics with sub-diffraction spatial resolution, STED microscopy has to be combined with a tool that is capable of capturing fast diffusion. Fluorescence correlation spectroscopy (FCS) is a commonly used microscope-based approach to probe the mobility of fluorescently labelled molecules ${ }^{8}$. In FCS, the autocorrelation function is calculated from the temporal fluctuations of the fluorescence intensity that are caused by transits of individual molecules through the microscope's observation volume (Figure 1b). The autocorrelation curve can provide information on the concentration and brightness of the diffusing molecules. Most importantly, it reveals the average transit time $\tau_{D}$ through the observation volume, from which the diffusion coefficient $D$ can be calculated (Eq. 3). For molecules diffusing in two-dimensions only, such as in lateral plane of biological membranes, the following relation is commonly used:

$$
D=\frac{d^{2}}{8 \ln 2 \tau_{D}}(\text { Eq. 3) }
$$

Here, a laser focus with a Gaussian lateral fluorescence excitation profile is assumed, and the beam diameter $d$ represents the effective observation spot size (full width at half maximum of the Gaussian profile), which can be extracted from calibration of the optical system as described below.

The diffusion coefficients that are accessible by FCS cover a broad range from $10^{3} \mu \mathrm{m}^{2} / \mathrm{s}$ for small molecules diffusing in solution, to $10^{-2} \mu \mathrm{m} / \mathrm{s}^{2}$ corresponding to large molecular clusters diffusing in densely packed environments. For detailed reviews on FCS, we refer the reader to ref ${ }^{3,9}$.

\section{Development of STED-FCS}


Spatially heterogeneous diffusion behaviour is often caused by molecular interactions that occur at spatial scales smaller than the resolution limit. Consequently, FCS performed on a conventional confocal microscope (i.e. with $d \approx 200 \mathrm{~nm}$ ) may miss this heterogeneity due to inherent averaging over the entire observation volume (Figure 1c). FCS can, however, be performed using subdiffraction-sized observation spots on a STED microscope. FCS data recorded for varying STEDlaser intensities yield values of the apparent diffusion coefficient $D$ (Eq. 3 ) as a function of the observation spot sizes. This dependence of diffusion coefficient $D$ on the observation diameter $d$, is generally referred to as the "diffusion law"10,11, and it reveals the heterogeneities in nanoscale diffusion (Figure 1d). For example, $D$ is independent of the size of the observation spot for a molecule that is undergoing free (Brownian) diffusion. For molecules that are undergoing transient immobilisation, $D$ decreases continuously with decreasing observation spot size ${ }^{12}$. For molecules that are transiently trapped in domains, $\mathrm{D}$ is initially decreased with a decreasing observation spot size but $\mathrm{D}$ levels out or slightly increases as the observation spot size gets closer to the diameter of the domain ${ }^{13}$. For molecules moving within a compartmentalized meshwork with partially impermeable barriers (hop or compartmentalized diffusion) ${ }^{14-16}, D$ increases towards smaller observation spot sizes ${ }^{13,17}$.

STED-FCS was conceptualised in $2005^{18}$, first applied to two-dimensional membrane diffusion measurements in live cells in $2009^{19}$, and has thereafter been extensively improved ${ }^{20-28}$. STEDFCS has two key advantages compared to confocal FCS; (i) the sub-diffraction-sized observation spots allows diffusion measurements on small structures $(<200 \mathrm{~nm})$ and (ii) the size of the effective observation volume can be tuned by the intensity of the STED laser, thus enabling direct determination of nanoscale diffusion law.

\section{Point STED-FCS and scanning STED-FCS}

The most common implementation of STED-FCS relies on fluorescence fluctuations measurements at a single stationary observation spot (point STED-FCS, pSTED-FCS) ${ }^{19}$. It can be performed with high spatial $(\approx 30 \mathrm{~nm})$ and temporal resolution $(\mu \mathrm{s})$. However, pSTED-FCS is limited by low statistics due to slow throughput (one FCS curve per measurement, typically taking 5-20 s each) as well as sparse spatial sampling (one spatial position per measurement). Therefore, 
it is challenging to investigate and adequately characterise highly heterogeneous structures such as the cellular plasma membrane ${ }^{29}$.

Recent adaptations of pSTED-FCS have overcome these caveats. Using fast laser beam scanners, scanning STED-FCS (sSTED-FCS) enables the acquisition of multiple STED-FCS curves along a line (or circle, usually a few micrometres long) with a high line-scanning frequency $(\sim 1-10 \mathrm{kHz})$. sSTED-FCS revealed spatial heterogeneity of lipid interactions in the plasma membrane with high statistical accuracy within short measurement times ${ }^{24}$. When combined with line-interleaved laser scanning (alternating STED and confocal laser illumination), sSTED-FCS can also directly resolve diffusion modes at multiple positions ${ }^{22}$. sSTED-FCS is therefore a very attractive tool particularly since it can be achieved in the classical imaging mode, available on any beam-scanning STED microscope ${ }^{21}$, (see Experimental Design “Available Systems” for details), i.e., without a need for dedicated equipment (such as a hardware correlator or single-photon counter). On the downside, temporal sampling of the correlation curve in sSTED-FCS, which is given by the line-scanning frequency, is reduced (typically to milliseconds compared to microseconds for pSTED-FCS). Consequently, fast diffusing molecules $\left(D>10 \mu \mathrm{m}^{2} / \mathrm{s}\right)$ cannot accurately be probed. Further, sSTED-FCS suffers from a reduced signal-to-noise ratio compared to pSTED-FCS; in pSTEDFCS, all the collected photons are used for one single curve, while for sSTED-FCS, it the acquisition time is split between multiple curves. This can be compensated for by longer acquisition times (30-90 s), where however potential bias due to cell movements, sample drifts etc. have to be more carefully considered and controlled. The spatial information of a STED-FCS measurement may further be increased by optimizing beam-scanning and data analysis, as done for spatio-temporal correlation ${ }^{25}$ and pair-correlation-function (pCF) analysis ${ }^{30}$ of line-scan data, or raster image correlation (RICS) analysis of raster-scanned images ${ }^{31}$. However these usually come at the expense of further reductions in the signal-to-noise ratio or lower spatial resolution.

In principle, two values of the apparent diffusion coefficient (one determined for the diffractionlimited confocal and the other for STED) are enough to determine the basic features of the diffusion mode ${ }^{12}$, yet detailed investigations (such as the difference between domain incorporation vs transient trapping, see Fig. 1d) require measurements of the diffusion coefficient $D$ for various observation spot sizes. For this purpose, a pulsed-interleaved excitation (PIE) scheme has been employed to acquire pSTED-FCS data for two or more STED laser intensities at a time ${ }^{28}$. 
Similarly, STED-FCS data and thus a diffusion law for multiple observation spot sizes can be generated out of a single recording with a continuous-wave STED laser in combination with pulsed excitation and software processing based on signal gating or lifetime filtering (STED-FLCS, fluorescence-lifetime correlation spectroscopy $)^{20,23}$.

The approaches presented so far were based on confinement of the observation spot along the lateral direction only (e.g. using the doughnut-shaped STED laser focus), which is sufficient to measure diffusion in two-dimensional samples such as membranes. However, a three-dimensional confinement of the observation spot might become necessary for measurements in solutions or in the cellular cytoplasm. 3-dimensional (3D)-STED-FCS is possible (see "Applications of the technique"), yet with decreased signal-to-noise level due to undepleted, uncorrelated, out-of-focus signal ${ }^{32}$. Methods reducing this uncorrelated signal have been introduced through improved fitting models ${ }^{26,32,33}$, optimized data analysis such as fluorescence lifetime based filters ${ }^{27}$, or dedicated microscope design and laser illumination schemes ${ }^{34}$. To keep this protocol concise, we restrict ourselves to the description of applying STED-FCS to the diffusion of molecules in a 2D plane, such as biological membranes, with the doughnut-shaped STED beam, providing the resolution improvement in the lateral direction.

\section{Overview of the procedure}

A schematic overview of the Procedure is shown in Figure 2. The procedure starts with culturing the cells of interest for one or two days including potential additional treatments such as transfections (Steps 1-3). On the day of the measurement, the STED microscope needs to be aligned and calibrated using fluorescent beads as well as supported lipid bilayers (SLBs) (Steps 410). This is followed by fluorescent labelling of the cells; it takes 5-10 minutes to incorporate fluorescent lipids into the plasma membrane (Step 11A), or $1 \mathrm{~h}$ if proteins are labelled via HaloSNAP tags (Step 11B; see Box 1). After labelling, the pSTED-FCS or sSTED-FCS measurements are performed (Step 12A and B respectively). While the basics of the pSTED-FCS and sSTED-FCS are very similar, they differ in the experimental procedure. Finally, the data is analysed using appropriate software (Step 13A and B respectively) (open-source versions are available, see Equipment). 


\section{Applications of the technique}

The small observation spot in STED-FCS has, for example, been exploited to measure the diffusion of protein molecules in the envelopes of single viruses and lipids in the membranes of small vesicles; measurements that would not be possible with confocal-based FCS ${ }^{35,36}$. The diffusion mode measurements with STED-FCS have yielded novel insights into molecular organisation of plasma membranes, shedding new light on the lipid-driven phase separation ${ }^{19,24,37,38}$ and the influence of the liquid-phase preference of lipid species and membrane proteins on their diffusion modes ${ }^{37,39-41}$. Moreover, STED-FCS has revealed how the cortical actin cytoskeleton $^{12,17,22}$, specific membrane proteins ${ }^{42}$, as well as the cholesterol content ${ }^{12}$ shape the cell membrane architecture, which in turn governs the hindered molecular diffusion modes of lipids and membrane proteins ${ }^{12,13}$. It has also allowed for the evaluation of diffusion characteristics of Ras kinase variants in tumour cells ${ }^{43}$, and of lipid-anchored proteins in mammalian cells ${ }^{22}$. Finally, STED-FCS has been useful to visualise the interactions between biomembranes and nanoparticles $^{44}$.

STED-FCS has also been employed for measuring molecular dynamics in 3-dimensions (3D) such as in cytoplasm and in solutions, for which also the axial depletion pattern can be used ${ }^{18}$. It has been used to probe the diffusion behaviour of cytoplasmic proteins, polymer mobility fluctuations, enzyme dynamics and binding-diffusion processes in solution ${ }^{27,45-47}$. Though the basic methodological principles are very similar, the optical setup and data analysis for accurate 3D measurements can be elaborate ${ }^{32}$, therefore rendering STED-FCS measurements of 3D diffusion is less established as of now.

\section{Comparison with other techniques}

Molecular diffusion modes in the plasma membrane are traditionally determined by single particle tracking (SPT) with mean square displacement (MSD) analysis. This approach relies on very bright and therefore often large fluorescent or scattering probes (such as quantum dots), fast acquisition and sparse label density ${ }^{48,49}$. STED-FCS does not require sparse labelling or bulky labels, bringing more experimental flexibility and less danger for potential probe-induced $\operatorname{artefacts}^{48,50}$. However, SPT gives unprecedented direct spatio-temporal information of individual molecules, while STED-FCS averages over an ensemble of molecules. These two approaches, therefore, may yield complementary information $37,50,51$. 
Spot-variation FCS (svFCS) introduced the framework for recording a diffusion law by obtaining and analysing FCS data for different observation spot sizes ${ }^{10,11,52,53}$. In contrast to STED-FCS, svFCS data is recorded for diameters larger than the diffraction-limit, thus allowing only for an indirect determination of nanoscale properties by extrapolation. Similar to svFCS, the concept of varying the observation spot size is also employed in other techniques mostly accessing larger (diffraction-limited) length scales. For instance, camera-based FCS variants or image-correlation approaches, where adjacent pixels are binned to obtain larger observation areas, are commonly used to gain insights into the diffusion behaviour ${ }^{54-56}$. Fast acquisition and image mean square displacement (iMSD) analysis directly discloses nanoscale hindered diffusion properties for short lag-times ${ }^{57,58}$. In these techniques, adjacent image pixels need to be integrated, thus they yield information over an average area. Notably, these approaches inherently provide spatial information and may even yield diffusion maps across the cells or directly reveal obstacles in the diffusion path for relatively slow-moving molecules ${ }^{59-61}$.

Near-field techniques avoid the requirement for lenses to focus light, but instead employ small apertures or plasmonic materials to directly access the same length and time scales as STEDFCS $^{62-64}$. However, the close proximity between the sample and optical elements impedes the broad applicability of these methods, for example, for exploring diffusion dynamics inside cells.

\section{Limitations of STED-FCS}

STED-FCS has historically been considered a complex technology that could only be realised in specialised labs with adapted STED microscopes. However, recent developments in commercial instrumentation have enhanced accessibility ${ }^{65}$ (discussed in Experimental Design "Available Systems"). Especially, sSTED-FCS can be employed without dedicated correlation hardware ${ }^{21}$. Nevertheless, applicability of STED-FCS is still more limited than of conventional FCS, since it usually requires fluorescent labels with a greater brightness and photostability, especially in cases where measurements at the smallest possible observation spot sizes are required. For example, while fluorescent proteins like GFP are commonly used in conventional FCS measurements, only very few STED-FCS studies have reported the use of GFP ${ }^{30}$. This is because fluorescent proteins, due to limited brightness and photostability, generally do not allow for measurements at observation spots below $\mathrm{d} \approx 80 \mathrm{~nm}$. These spot sizes are far larger than those which can be achieved using organic dye labels for example through live-cell protein labelling approaches using e.g. 
SNAP and Halo epitope tags (Box 1). Further discussion on available probes is provided in "Fluorescent probes for STED-FCS" below. Furthermore, as highlighted before, STED-FCS applications have thus far mainly been restricted to two-dimensional diffusion measurements in membranes, and application to three-dimensional diffusion problems still require further improvement in analysis and acquisition protocols. Finally, spatial information of STED-FCS is limited, so far giving simultaneous access to nanoscale diffusion properties along a line only (sSTED-FCS). Future developments might therefore involve an optimized combination of imagecorrelation approaches on a STED microscope as has been indicated for RICS ${ }^{31}$ or predicted for $\mathrm{iMSD}^{58}$.

\section{Expertise needed to implement the protocol}

To prepare the calibration samples, the researcher has to be familiar with common wet-lab techniques. Cell experiments require basic expertise in cell culturing, including transfection if fluorescent proteins are to be expressed. To successfully perform STED-FCS experiments, the researcher should be trained in basic operation of the STED microscope. Depending on the design of the particular setup (e.g. commercial turn-key instrument vs home-built open-optics setup), this may necessitate different levels of familiarity with basic principles of optics. Often, the local rules may require the operator to undertake laser safety training. In any case, a good technical understanding of the instrumentation helps with optimization of the measurement parameters and with troubleshooting. The skills in data analysis and interpretation come with experience and initial experiments should therefore be performed on robust control samples (e.g. SLBs) in order for a researcher to obtain the required confidence with the sample preparation, data measurement and analysis steps.

\section{Experimental design}

Fluorescent probes for STED-FCS. Several issues have to be considered for an accurate choice of fluorescent probes for STED-FCS measurements. First, the fluorophore should not absorb at the wavelength of the STED depletion laser. This is because the required power of the STED laser vastly exceeds conventional excitation lasers such that even a small overlap of the STED laser wavelength and the excitation spectrum of the fluorophore will lead to rapid photobleaching. Therefore, the choice of the dye has to match the available instrumentation. i) The fluorophores 
should be very photostable and bright, emitting as many photons as possible during their transits through the effective observation spot in order to produce an accurate correlation function at sufficient signal to noise. Second, fluorescent dyes with long fluorescence lifetimes $(\sim 4 \mathrm{~ns})$ tend to work conveniently with $\mathrm{STED}^{66}$, in particular in the cases of time gated STED-FCS with a CW STED laser. Another point to consider is the excited state photophysics of the chosen fluorophores. Some dyes (or fluorescent proteins such as GFP) may experience altered photophysical properties in the excited state and in some cases this may result in the absorption of the STED light and thus leading to rapid bleaching ${ }^{67}$. This may be avoided by checking for the excited-state absorption or by fast laser-beam scanning (as for sSTED-FCS) in the case of triplet or dark state absorption.

There are several commercially available organic dyes (such as Atto and Abberior fluorophores, see Table 1) that fulfil the above criteria and which hence perform well in STED-FCS in our hands. Also, new organic dyes potentially compatible with STED-FCS are constantly being developed ${ }^{68}$.

Robust and artefact-free labelling of lipids and proteins with the organic fluorophores is one of the biggest challenges in fluorescence microscopy, since a bias in biochemical characteristics due to the modification can never be excluded. For lipids, this is particularly problematic due to the comparable size of lipids and fluorescent tags. The behaviour of the fluorescent lipid analogues depends strongly on the choice of both the native lipid and the fluorescent tag, as well as on the labelling strategy ${ }^{37,69}$. In general, the least perturbing strategy is to attach the fluorophore to the headgroup rather than to the acyl chain, and further by insertion of a hydrophilic linker between the headgroup and the fluorophore to ensure that the fluorophores (which may react with membranes $^{70}$ ) are well separated from the lipid headgroups ${ }^{69}$. Nevertheless, thorough controls have to be performed to validate the extent to which the fluorescent lipid analogues preserve the natural physicochemical behaviour of the native lipid.

The most common way to fluorescently tag proteins of interest uses genetic fusion with fluorescent proteins. A few fluorescent proteins (such as mCitrine, mVenus etc) can be used with STED imaging $^{71}$, however, they generally cannot be used at high STED powers and therefore do not yield as high spatial resolution as organic dyes. This limits their use in STED-FCS ${ }^{19,30}$. To overcome this, there is a substantial effort to develop brighter and more photostable fluorescent proteins. mGarnet $^{72}$, E2-Crimson ${ }^{73}$ and TagRFP657 $7^{74}$ are, for instance, a few recent fluorescent proteins whose use for STED imaging in live cells has been demonstrated. Furthermore, novel technologies 
have emerged to label proteins with bright and photostable organic dyes using Halo or SNAP $\operatorname{tags}^{75,76}$.

It is important to note that not all dyes that perform well in STED imaging will necessarily work as well for STED-FCS, such as Oregon Green $488^{20,77}$, such that further testing of new fluorophores is always advisable. Please see Table 1 for a collection of STED-FCS suitable fluorophores and Box 1 for more information on the probe selection.

Available systems. To the best of our knowledge, there are five vendors of STED microscopes on the market (Abberior Instruments, ISS, Leica Microsystems, Lumicks, and PicoQuant), all of which can perform STED-FCS experiments via one of the modalities mentioned below. There are three approaches for measuring STED-FCS on a STED-capable microscope (main differences summarised in Table 2, and discussed in detail below):

1- Time mode $e^{22,24,25,30,78}$ : Data recordings in this mode consist of an intensity time trace (i.e. number of photons detected within consecutive time intervals) as a 1D ( $t$-scan) or $2 \mathrm{D}(x t$ scan) data set for pSTED-FCS and sSTED-FCS, respectively. This data is subsequently further software-processed to yield FCS curves.

2- Correlator-based ${ }^{13,18,19}$ : Data recordings in this mode uses an analogue correlator to calculate the FCS curves on the fly (during the measurement) directly from the detected intensity signal. This modality is only possible for pSTED-FCS measurements.

3- Photon mode $20,23,25,27$ : Data recordings in this mode uses time-correlated single-photon counting (TCSPC) electronics to record the arrival times for each detected photon- both from the beginning of the experiment (commonly referred to as "time-tagged" or "macrotime") and from the previous excitation pulse ("time-resolved" or "micro-time"). Appropriate software is then used to convert the photon streams into FCS curves. This modality most commonly measures pSTED-FCS but can also be used for sSTED-FCS.

All STED microscopes, including home-built setups, can perform the time-mode pSTED-FCS experiments (Mode 1) out of the box. The 2D (xt) scans for sSTED-FCS require a mirror beam scanner (typically integrated in STED imaging microscopes) rather than a piezo objective scanner to provide sufficient sampling frequency. It allows for high photon count rates (up to $30 \mathrm{MHz}$, defined primarily by the dead time of the detectors). If supported by detection electronics, timegating can be set before acquisition to remove the contributions of residual confocal fluorescence 
and thereby improve spatial resolution. On the downside, no preview into the resulting FCS curve is possible while acquiring the data, which would inform the experienced user about an occurrence of unwanted events (see "Controls and artefacts") and hence increase the efficiency of the workflow. However, this mode allows for post-acquisition cropping of the time trace to remove such events and restore the relevant information ${ }^{21}$. Perhaps the main drawback is related to its temporal resolution, which is limited by the line scanning frequency (typically $1-10 \mathrm{kHz}$ ).

Correlator-based STED-FCS (Mode 2) requires additional hardware, specifically an analogue correlator, which is commercially available (e.g. ALV, correlator.com, etc.). Advantages of this approach are: (i) the correlation curves are calculated on the fly (i.e. no post-processing of the detected signal is necessary) and data can be directly visualised during acquisition; (ii) the output files are small (usually a few kB only) and can be straightforwardly analysed using custom-written free software ${ }^{79,80}$. Disadvantages of this method are: (i) the maximum photon count-rate is limited to a few $\mathrm{MHz}$, which is defined by the internal clock frequency of the hardware correlator, and, (ii) the data cannot be post-processed to correct for possible bias and artefacts.

The photon mode (Mode 3) offers the highest post-processing flexibility (such as time gating and cropping of the signal), and the highest time resolution capable of capturing very fast dynamics. Disadvantages are; (i) it requires additional TSCPC hardware, (ii) the data processing is more complex usually requiring specific vendor-provided or custom-built ${ }^{21}$ software, (iii) higher computational power needed for calculating the correlation curve, which can take several minutes, and (iv) the resulting output files are the largest, necessitating more attention on data-management (e.g. disk space, file transfer and sharing). Very recent advances in hardware development alleviated another previous restriction - the maximal detected photon count-rate was limited to few $\mathrm{MHz}$, which can now be greatly exceeded [refs].

Alignment of the optical system. Before starting any STED-FCS measurements, the experimental apparatus, either commercial or custom, has to be properly aligned and calibrated. First, alignment between the excitation and depletion beams must be assured in space (Figure 3a,b and Steps 6-8) and time (Figure 3c, Step 9), yielding the highest signal levels at the best possible spatial resolution.

The foci of the excitation and (doughnut-shaped) STED lasers have to be accurately aligned, i.e. perfectly overlaid in space. On some commercial STED microscopes (e.g. Leica), the spatial 
alignment is achieved by running a built-in automatized routine. Otherwise, this procedure requires a manual optical adjustment (Steps 6-8 of the Procedure). In order to perform such manual alignment, immobilised fluorescent beads (e.g., fluorospheres; yellow-green, red and crimson beads, for green, orange and red spectral regions, respectively) of a small size $(\approx 25-40 \mathrm{~nm}$ in diameter) can be utilized. Comparing the confocal and STED images of the same area of the calibration sample, a concentric overlap of the two images should be obtained (Figure 3a), confirming a proper co-alignment of the two beams (Figure 3b).

Once this very first step has been completed, the temporal alignment needs to be optimised (to our knowledge, none of the commercial instruments performs this step automatically) (Step 9 of the Procedure), which can be performed via STED imaging of the very same sample as above (Step 9A). By adjusting the timing settings for either the gating (in case of CW STED laser) or the delay between the excitation and STED laser pulses (in case of pulsed STED laser), a sharp STED image should be obtained with negligible confocal contribution that otherwise surrounds the signal of each super-resolved bead (Figure 3c, Step 9A). Alternatively, the optimal temporal synchronisation can be achieved by minimising the intensity of fluorescent probes diffusing in an SLB while acquiring rapid STED images or a time trace from a single point (Step 9B).

Calibration of the observation volume of the optical system. After alignment, the sizes of the observation spots at different STED powers have to be calibrated, which is necessary for accurate determination of diffusion coefficients later on. To calculate the lateral diffusion coefficients $(D)$ from the empirical transit times $\left(\tau_{\mathrm{D}}\right)$ extracted from fitting the correlation curves (see Eq. 3 ), the diameters of the effective observation areas $(d)$ for each STED power are needed. This calibration can be performed with either imaging (Step 10 A) or FCS mode (Step 10B and C).

The diameters of observation spots in confocal $\left(d_{C O N F}\right)$ and STED conditions $\left(d_{S T E D}\right)$ can be measured from images of the aforementioned calibration sample with immobilised fluorescent beads, by fitting the intensity profile across the beads with a Gaussian profile and extracting the full width at half maximum (FWHM) (Figure 3b). The average of the FWHM of many (at least 10-30) measured profiles across isolated single beads provides a good estimate of $d$ (Step 10A). This can be done manually using the ImageJ/Fiji Curve Fit functionality, or with freely available plugins, such as $\mathrm{PSFj}^{81}$. 
The second approach is based on FCS measurements of a freely diffusing dye (e.g. a fluorescent lipid analogue in a supported lipid bilayer, Figure 3d) with a priori knowledge of the characteristic diffusion coefficient at the measurement conditions (Step 10B for pSTED-FCS and 10C for sSTED-FCS). $d_{C}$ can be calculated, knowing $D$ and deducing $\tau_{D}$ from the confocal FCS curves. Owing to the free diffusion of the dye, i.e. the diffusion coefficient being independent of the observation spot size, the observation spot diameter for each STED power can be calculated via:

$d_{S T E D, i}=d_{C O N F} \sqrt{\frac{\tau_{D, S T E D, i}}{\tau_{D, C O N F}}}$ (Eq. 4$)$

$d_{S T E D, i}$ is the apparent observation spot size for given STED laser intensity $i$ and $\tau_{D, C O N F}$ and $\tau_{D, S T E D, i}$ are determined by averaging fit results (described later) of correlation curves acquired in confocal and at various STED powers. The acquisition of a series of STED-FCS measurements at increasing STED laser powers will yield autocorrelation curves shifted towards faster time scales (Figure 3e) and smaller observation spot diameters (Figure 3f).

This calibration enables the diffusion coefficient $D$ to be calculated from any diffusion time $\tau_{\mathrm{D}}$, providing the molecular diffusion law. The described imaging and FCS based calibration approaches (Steps 10A and B, C) yield comparable results for the commonly used alignment/calibration samples (such as Crimson beads) and best-performing STED dyes (such as Atto647N and Abberior Star Red) ${ }^{19}$ and are therefore often combined ( $d_{C O N F}$ determined by imaging, $d_{S T E D, i}$ with FCS). However, other dyes may have vastly different depletion efficiencies (e.g., due to different fluorescence lifetimes), resulting in non-comparable effective observation spot sizes under the same conditions ${ }^{51}$. To assure reliable determination of $D$ and diffusion modes, it is therefore essential to conduct the calibration with a similarly performing dye and under similar experimental conditions as the final experiment (e.g. powers of the excitation and STED lasers, temperature). For sSTED-FCS in particular, the calibration should be performed in the scanning mode.

Measuring the mobility of molecules in membranes of live cells. After calibration of the microscope and labelling of the sample (Step 11), STED-FCS measurements on cells can be performed (Step 12). As with classical imaging and FCS, a few practical points need to be considered when designing the acquisition scheme. 
- If the experiment is performed at any other than room temperature (e.g. physiological), the sample and sample chamber should be allowed to fully equilibrate for at least 1015 minutes after placement onto the microscope stage to avoid focus z-drift. In this case, the alignment and calibration should also be performed at the chosen temperature.

- Extreme care should be taken to ensure that the coverslips with the adherent cells are mounted straight as any tilting of the coverslip can cause artefacts. The laser spot should be focussed perfectly on the membrane, which can be achieved by moving the focus up and down and maximising the fluorescence intensity. Perfect focusing will yield the FCS curves with the shortest transit times and highest amplitudes, although this is a bit less critical in 2D STED-FCS when compared to confocal FCS ${ }^{36}$.

- $\quad$ Large, bright and immobile structures should be avoided, unless those are the structures of interest, as they will often show poor STED effect or significant bleaching, which can considerably alter the readout ${ }^{21}$.

- The acquisition time should be kept constant for the course of the experiments; it should be sufficiently long (typically 10-20 s for pSTED-FCS and 10-90 seconds for sSTEDFCS) to result in adequate signal-to-noise ratio of the curves and to capture the slow diffusion, yet short enough to avoid excessive bleaching, and phototoxicity.

- $\quad$ The average fluorescence intensity should not fluctuate drastically during acquisitions. These types of fluctuation can be caused by photobleaching, bright clusters (such as internal vesicles etc.) and they will dominate and bias the correlation.

- Multiple data recording repetitions should be performed at one location with randomly varied STED laser powers (ascending and descending order) to avoid potential STEDinduced artefacts.

- The chosen excitation power should be sufficiently low (typically up to $10 \mu \mathrm{W}$ for farred STED-FCS dyes) to avoid fluorophore saturation, excessive photobleaching, and overflow of the detection electronics.

- $\quad$ For sSTED-FCS, in addition, the scanning parameters have to be optimised to achieve a decent signal-to-noise ratio at the required spatial and temporal resolution. To ensure adequate spatial sampling, the pixel size should be half of the diameter of the smallest anticipated observation spot obtained at the corresponding STED laser powers. Temporal sampling, defined by the line scanning frequency, should be at least 
comparable to the fastest expected transit times (these can first be measured with pSTED-FCS at similar spatial resolution), but preferably a few times faster to assure reliable analysis. The total acquisition time needs to be significantly longer than for pSTED-FCS, but short enough to avoid artefacts, due to cell movement or sample drifts (typically 10-90 s). Moreover, the length of the scanning line and pixel dwell time have to be balanced.

- If the data are acquired in the time-mode (as $x t$-scan images), time-gating of the detection should be set to clean-up the scattered and confocal contributions to the signal, arriving shortly after the laser pulses.

Data analysis. The analysis workflow is primarily dependent on the applied technical approach in the acquisition of STED-FCS data (i.e., time, correlator, or photon modes; see Table 2) as each mode yields data in its specific format. For clarity, however, we here discuss the analysis steps in the context of spatial sampling; single-point (Step 12A) and line-scanning (Step 12B). These are directly supported with the two freely available software packages used here (FoCuS-point and FoCuS-scan, see Equipment). pSTED-FCS (single point) data are acquired as correlated or TCSPC data (Table 2). The correlated data are ready for fitting without any further analysis step. On the other hand, TSCPC data are organised such that the arrival times of individual photons are logged on the macro and micro time-scales (i.e., the total acquisition time and the time from the last excitation pulse, respectively), which requires additional pre-analysis steps. The recorded signal is often contaminated by a non-negligible amount of scattered laser light or fluorescence originating from outside the STED observation volume, especially when a continuous-wave depletion laser is applied $^{20}$. Given the knowledge of the micro arrival time of the emission photons relative to the laser excitation, this artefact can be removed through employing a micro time-gated filtering scheme prior to the correlation of the signal on the macro time-scale $20,23,82$. To this end, pieces of software exist that utilise correlation algorithms designed specifically for TSCPC data as well as for time-gating analysis $79,80,83,84$.

In sSTED-FCS, which involves the simultaneous scanning of the excitation and STED lasers over a line or circular region repetitively for the duration of the experiment ${ }^{24}$, photons are typically integrated, resulting in 2D ( $x t$-scan) images. The intensity trace from each spatial pixel in the line 
is then correlated individually. For such sSTED-FCS measurements, time-gating can only be applied during the acquisition. Prior to correlation, the time traces can be cropped to exclude unwanted regions (e.g. signal from large particles passing the laser beam, or initial seconds dominated by photobleaching of immobile fluorescent probes). In addition, the gradual decay of the fluorescence intensity due to photobleaching of mobile molecules, which can cause significant distortions to the resulting correlation curves and bias to the extracted FCS parameters, can be corrected for ${ }^{21,36,85}$.

In this protocol, we will describe how to perform analysis of STED-FCS data using freely available FoCuS-point ${ }^{79}$ and FoCuS-scan ${ }^{21}$ software for pSTED-FCS and sSTED-FCS respectively, although the principles are equally valid for alternative correlation software. FoCuS-point software utilizes a fast TSCPC correlation algorithm ${ }^{83}$ and also contains tools for time-gating of photons based on their micro arrival times, before subsequent macro-level correlation. FoCuS-scan software employs a fast multi-tau correlation algorithm and includes tools for photobleaching corrections. Both pieces of software support batch analysis with a variety of fitting models to accommodate different diffusion dynamics ${ }^{79}$, and include methods for data export at any stage of the analysis process.

Controls and artefacts. Typical artefacts of confocal $\mathrm{FCS}^{86,87}$ constitute a problem also for STEDFCS (also see Anticipated Results for examples). First of all, general stability of hardware and electronics is absolutely essential for robust STED-FCS measurements. For instance, each experiment should be tested for focus drift, particularly in between measurements. If the laser spot is not perfectly focused on the membrane, the transit times will be biased towards longer time scales $^{52}$. Similar effects can be anticipated from axial drift of the sample (e.g., good thermal equilibration of the sample on the temperature-controlled stage is key) or inconsistent temporal synchronisation of electronics signals (warming-up of the equipment before starting any measurements is essential).

The most common sample-related artefact of STED-FCS measurements arise from photobleaching of the fluorescent dyes, which affects the shape of the auto-correlation curve leading to a miscalculation of the diffusion times. In the most extreme case, when a large fraction of the transit times of the molecule through the excitation spot are subject to the fluorophore degradation, this 
can result in a shortening of the transit time ${ }^{20}$. Otherwise, the gradual decay of the average intensity results in a pronounced offset of the correlation curve, resulting in erroneously prolonged transit times $^{21}$. This can, to some extent, be mitigated using bleaching-correction algorithms ${ }^{85}$, unless the data were acquired in the correlation mode (those measurements should be discarded or interpreted very carefully). If the photobleaching is due to immobile fluorophores, the area of interest can be pre-bleached or the initial part of the time trace can be cropped out. Nevertheless, if possible, photobleaching should be prevented by choosing a more photostable dye or by decreasing the power of the excitation (and STED) laser.

Another common issue results from very bright particles, such as molecular clusters or vesicles, occasionally moving in and out of the focal volume, which will dominate the shape of the correlation curves and typically shift them towards longer transit times and higher FCS amplitudes. While these events can sometimes be identified and cropped out from the acquired time-traces (sometimes referred to as "spike-filtering"), no correction is possible for such data recorded with the hardware correlator, which should therefore be immediately discarded. In that mode, it may thus be useful to run a larger number of shorter measurements, which will increase the chances of acquiring some non-affected data.

As STED-FCS often requires rather long measurements at the same position (several recordings at multiple STED powers, or minute-scaled $x t$-scans), one needs to assure that the diffusion properties have not changed during the acquisition. It is therefore advised that confocal measurements are repeated after each STED recording, which should in turn be performed in both ascending and descending order of the STED power.

Specific to STED-FCS, it is crucial to have a reliable free-diffusion calibration sample as control for every experiment, the comparison to which will make it possible to recognise any real hindrance of diffusion and attribute it to true interactions. Such calibration conducted in cells with a supposedly freely diffusing membrane probe, may not be reliable, as their diffusion behaviour can vary between different cell types. So far, only cholesterol analogues have been shown to diffuse freely consistently with different techniques ${ }^{24,41,88}$. Nevertheless, measurements of freely diffusing fluorescent phospholipid analogues in model membranes (e.g. SLBs) composed of liquid-disordered phase lipids (such as DOPC) should precede every experiment with cells. 


\section{MATERIALS}

\section{Biological Materials}

- Cells of interest: Any cell line can be used. Here we use PtK2 cells (ATCC, no. CCL$56^{\mathrm{TM}}$ ), but our approach can be used in combination with any cell line or primary cell culture of interest.

CAUTION: Cell lines should be contamination- and mycoplasma-free.

\section{Reagents}

\section{Cell culture}

- DMEM (Invitrogen, cat. no. 21090-022),

- Fetal bovine serum (Sigma-Aldrich, cat.no 12103C),

- L-glutamine (Life Technologies, cat. no. 25030081)

- Leibovitz L15 medium without phenol red (Thermo Fisher, cat. no. 21083027)

\section{Lipids}

- 1,2-dioleoyl-sn-glycero-3-phosphocholine (DOPC; Avanti, cat. no. 850375P)

- Cholesterol (Avanti, cat. no. 700000)

- Abberior Star Red labelled phospholipid (Abberior, cat. no. 2-0311-011-7)

- Abberior Star Red labelled cholesterol with PEG linker (Abberior, custom synthesis)

- Atto647N-labelled 1,2-Dipalmitoyl-sn-glycero-3-phosphoethanolamine (AttoTec, cat. no. AD 647N-151)

- Atto647N-labelled sphingomyelin (AttoTec, custom synthesis)

- Methanol (as lipid solvent) (Sigma-Aldrich, cat. no. 322415)

- Chloroform (as lipid solvent) (Sigma-Aldrich, cat. no. C2432).

CAUTION: Chloroform is toxic when inhaled, thus it is recommended to work in a fume hood when working with chloroform.

\section{Protein Labelling}


- SNAP-Cell 647 SiR, (NEB, cat no. S9102S)

- Abberior Star BG (Abberior, custom synthesis)

- HaloTag TMR (Promega, cat no. G8251)

\section{Buffers}

- $\mathrm{NaCl}$ (Sigma-Aldrich, cat. no. S5886)

- $\mathrm{KCl}$ (Sigma-Aldrich, cat. no. P5405)

- $\mathrm{HCl}$ (Sigma-Aldrich, cat. no. H1758)

- Sulfuric acid, 99.99\% (vol/vol) (Sigma-Aldrich, cat. no. 339741)

- Hydrogen peroxide, 30\% (wt/wt) in H2O (Sigma-Aldrich, cat. no. H1009)

- PBS (pH 7.4; Gibco, cat. no. 10010023).

- Mowiol (Sigma-Aldrich, cat. no. 81381).

- Poly-L-Lysine (Sigma-Aldrich, cat. no. P4707)

CAUTION: Sulfuric acid is highly corrosive, thus handling of sulfuric acid should be done in a fume hood with protective gloves.

\section{Plasmids and transfections}

- GPI-SNAP plasmid (from Stefan Hell Lab, Goettingen, Germany)

- Lipofectamine 3000 transfection reagent (ThermoFisher, cat. no. L3000015)

- Opti-MEM I reduced serum medium (ThermoFisher, cat. no. 11058021)

- Trypsin-EDTA $(0.25 \%$ (wt/vol); ThermoFisher, cat. no. 15050065$)$

\section{Equipment}

- STED microscope with 100X Oil (NA=1.4) objective and $37^{\circ} \mathrm{C}$ chamber (see Available Systems for the options). CRITICAL Before starting the procedure, measure your STED beam laser power to determine the actual laser power on the sample with varying power percentage.

- Glass coverslips (no. 1.5, $25 \mathrm{~mm}$ round; SLS, cat. no. MIC3350)

- Metal holder for $25 \mathrm{~mm}$ round coverslips (Attofluor ${ }^{\mathrm{TM}}$ Cell Chamber, ThermoFisher, cat.no. A7816)

- T25 culture flasks (Corning, cat. no. 430639) 
- Biosafety cabinet (CAS, model no. Biomat-2)

- Cell culture incubator (Panasonic, cat. no. MCO-170AIC-PE)

- Glass bottom dishes (no. 1.5; Ibidi, cat. no. 81158)

- Parafilm (Sigma-Aldrich, cat. no. P7793-1EA)

- Amber glass $2 \mathrm{ml}$ vials for lipid storage (manufacturer Supelco, supplier Sigma-Aldrich, cat. no. 27083-U)

- Caps for $2 \mathrm{ml}$ vials with PTFE liner (Sigma-Aldrich, cat. no. 27091-U)

- FluoSpheres, crimson (625/645) diameter $0.02 \mu \mathrm{m}$ (ThermoFisher, cat. no. F8782)

- Glass pasteur pipette (VWR, cat. no. 612-1701)

- $1.5 \mathrm{ml}$ tubes (Eppendorf, cat.no. 22431021)

- Glass beakers

- Safety Spectacles

- Protective gloves

- Optional: FCS Correlator Card (correlator.com)

\section{Software}

- FoCuS-point software ${ }^{79}$ and manual (freely available at https://github.com/dwaithe/FCS_point_correlator) (release DOI:10.5281/zenodo.1314184)

- FoCuS-scan software ${ }^{21}$ and manual (freely available at https://github.com/dwaithe/FCS_scanning_correlator) (release DOI:10.5281/zenodo.1292334)

- ImageJ/Fiji ${ }^{89}$ (freely available at https://imagej.net/Fiji/Downloads)

- For correlation and fitting of FCS data, alternative software packages are widely available such as $\mathrm{PyCorrFit}{ }^{80}$ or $\operatorname{simFCS}{ }^{84}$.

\section{Reagents Setup}

Cell Growth Medium. Prepare cell growth medium according to cell-specific instructions at ATCC. Here, we use PtK2 cells. Supplement high glucose DMEM with 15\% (vol/vol) FBS and $1 \%$ (vol/vol) L-glutamine. This can be stored at $4{ }^{\circ} \mathrm{C}$ for $2-3$ months.

Piranha-cleaned coverslips. Mix $\mathrm{H}_{2} \mathrm{SO}_{4}$ and $\mathrm{H}_{2} \mathrm{O}_{2}$ with 3:1 (vol:vol) ratio in a glass beaker. Pour $\mathrm{H}_{2} \mathrm{SO}_{4}$ first and add $\mathrm{H}_{2} \mathrm{O}_{2}$ slowly. Piranha solution must be prepared fresh and must not be stored. Put coverslips into the piranha solution. Make sure that there are no bubbles preventing the 
thorough exposure of coverslips to the piranha solution. The mixture can gently be mixed with a glass Pasteur pipette. Do not use pyrex pipettes. Incubate for 30-45 minutes, stir occasionally. Wash the glass slides carefully by pouring big volume of MilliQ water $(>2 \mathrm{~L})$ in the same container. Make sure that all the piranha solution is washed out. Leave glass coverslips inside MilliQ water. The coverslips can be used for a few days after cleaning.

CAUTION: Piranha solution is highly corrosive. Wear protective gloves and safety spectacles. Work in the fume hood, do not take piranha solution out of the hood. NEVER put Piranha solution in closed containers as explosions can occur due to gas generation and over-pressurization. Use always glass containers. Explosions may also occur when Piranha reacts with organic material or if the peroxide solution concentration is more than $50 \%$ ( $\mathrm{vol} / \mathrm{vol})$. Therefore the peroxide must ALWAYS be added to the sulphuric acid, NEVER vice versa. Please consult with your local safety officer before preparing and using Piranha solution.

Fluorescent Lipid Solutions. The solid dyes are dissolved in ethanol. The stock solutions are optimally $0.5 \mathrm{mg} / \mathrm{ml}$. Stock solutions should be kept in lightproof vials at $-20^{\circ} \mathrm{C}$. The vials should be properly sealed and can be stored for a year.

CRITICAL: To avoid oxidation the air should be removed from the vials before storing the stocks. The best strategy is to replace air with a noble gas such as nitrogen or argon. If the air in the vials is removed and the lightproof vials are properly sealed, the stock solutions can be stored for a year.

SLB Buffer. Prepare $10 \mathrm{mM}$ HEPES and $150 \mathrm{mM} \mathrm{NaCl}$ at $\mathrm{pH}$ 7.4. If sterilised, this buffer can be stored for 2 months at room temperature.

Transfection of the cells. If protein dynamics are to be measured, cells should be transfected beforehand. For PtK2 cells, Lipofectamine 3000 yields $40-50 \%$ transfection efficiency. Cells need to be seeded on glass bottom dishes or coverslips two days before the transfection. For each well of a 6-well plate, $4 \mu \mathrm{L}$ of L3000 and $5 \mu \mathrm{L}$ of P3000 should be used. In one tube, mix $125 \mu \mathrm{L}$ of OptiMEM with $500 \mathrm{ng}$ DNA and $5 \mu \mathrm{L}$ of P3000. In another Eppendorf tube, mix $4 \mathrm{uL}$ L3000 with $125 \mathrm{uL}$ OptiMEM. Mix the contents of both vials and incubate for at least 15 minutes. After transferring the solution onto the cells, incubate the transfection for about 4 hours and replace the medium with fresh culture medium afterwards. Keep transfection solutions in the fridge till the expiration date. 
Preparation of beads sample for alignment and calibration. Dilute fluorescent microspheres (e.g. FluoSpheres, crimson (625/645) diameter $0.02 \mu \mathrm{m}$, Invitrogen, USA) 1:10 (vol:vol) in Milli-Qwater. Depending on the stock solution the dilution might need to be optimized. Put a $100 \mu \mathrm{l}$ drop of poly-L-lysine on a microscope coverslip and let it rest for 5 minutes. Rinse gently the poly-Llysine with Milli-Q water. In the same coverslip area add a $100 \mu \mathrm{l}$ a drop of the diluted beads solution and let it rest for other 5 minutes. Wash gently with Milli-Q water. Mount the coverslip (beads face down) on a microscope slide, embedding in the mounting medium Mowiol. Seal the coverslip to the slide. This sample can be stored at $4^{\circ} \mathrm{C}$ a light proof container and used for few months. The sample is degraded when the beads start to break releasing the fluorescent dye. As a result the beads look less defined in shape and the signal/noise ratio decreases. The desired sample will show distinct bright beads all over the field of view.

Preparation of SLBs for alignment and calibration. Supported lipid bilayers (SLBs) can be prepared by spin-coating of a lipid solution onto a hydrophilic coverslip: First dissolve lipids (DOPC for pSTED-FCS, or DOPC with up to $50 \mathrm{~mol} \%$ of cholesterol for sSTED-FCS) to a total concentration of $1 \mathrm{mg} / \mathrm{mL}$ in a 1:1 (vol:vol) mixture of chloroform and methanol. Dope the mixture with a small amount of fluorescent lipid (e.g. DPPE-Abberior STAR Red) at a molar ratio of about 1:5000 - 1:10000. Drop $25 \mu \mathrm{L}$ of the lipid solution onto a dry piranha cleaned $25 \mathrm{~mm}$ round coverslip ( $0.17 \mathrm{~mm}$ thick) (see Reagent Setup) and spin coat it at $3000 \mathrm{rpm}$ for 30 seconds. Place the coverslip onto the metal chamber. Hydrate the lipid film promptly by adding $1 \mathrm{~mL}$ SLB buffer. If required, carefully wash the SLB by adding and removing more buffer a few times while ensuring that the SLB is not drying as this destroys the bilayer.

CAUTION: Care should be taken when handling these carcinogenic substances (e.g., chloroform and methanol). If possible handle all lipid solutions and perform spin coating in a fume hood. CRITICAL STEP: Store lipids in amber glass vials at $-20^{\circ} \mathrm{C}$. Lipids can be oxidised when exposed to air for a long period time. Try to keep the lipids outside of the freezer as short as possible. Replace the air with Argon or Nitrogen after using the lipids by shortly blowing the vial with these gases. After this step, seal the cap of the vial tightly with parafilm to avoid any evaporation as well as oxygen flow into the tube.

CRITICAL STEP: Start spinning as soon as dropping the solution on the coverslip, otherwise multilamellar bilayers will be formed. 
CRITICAL STEP: STED depletion will naturally decrease the signal thus requires higher labelling. 1:5000 molar ratio is sufficiently low to enable confocal FCS data recordings and sufficiently high for STED-FCS data recordings.

\section{PROCEDURE}

\section{Preparation of cells. Timing: 1-2 days}

1. Seed the cells of interest in an appropriate chamber. When protein dynamics are to be measured, transfect the cells as described in the Reagent Setup.

CRITICAL STEP: Further steps in the protocol are optimized for cells seeded on glass bottom 35-mm cell culture dish. Our example applies for adherent cells but can also be used (adapted) for suspension cells (labelled in suspension, and then dropped on a cover slip).

CRITICAL STEP: Seed the cells on $0.17 \mathrm{~mm}$ (\#1.5) glass slide for the best results.

2. Incubate the chambers in appropriate conditions until cells reach $\sim 50-70 \%$ confluency.

3. Optional: Treat the cell culture (e.g. transfection, drug exposure, nutrient starvation etc.) depending on the biological question. If you would like to investigate proteins, please refer to BOX 1 for best protein labelling strategies.

\section{Preparation of samples for alignment and calibration. Timing: $30 \mathrm{~min}$}

4. Generate a beads sample as described in "Preparation of beads sample for alignment and calibration" (Reagent Setup).

5. Prepare the SLB sample as described in "Preparation of SLBs for alignment and calibration" (Reagent Setup).

\section{Alignment and calibration of the system before the cell measurements. Timing: $1 \mathrm{~h}$}

CRITICAL: The alignment and calibration steps should be done every day before starting the measurement. See Experimental Design "Alignment of the optical system" for detailed information on the different alignment strategies.

\section{Spatial alignment of the excitation and STED lasers (Steps 6-8):}

6. Place the beads sample (see Step 4) onto the microscope carefully.

7. Take an image in confocal and high power STED mode.

8. Overlay the images to test if they are perfectly superimposed.

CRITICAL STEP: Make sure the beads are exactly in focus (use xz or yz scan to check) and if the slide was stored at $4{ }^{\circ} \mathrm{C}$ let it equilibrate to measurement temperature prior to alignment. 


\section{TROUBLESHOOTING}

\section{Temporal alignment of the excitation and STED lasers (Steps 9):}

9. For temporal alignment in imaging mode using beads sample follow Option A, or FCS mode using SLBs, follow Option B.

Option A: Take images of the same beads sample in high power STED mode and vary the temporal delay between excitation and STED pulses till you achieve the sharpest image .

Option B: Perform FCS on the SLB sample in high power STED mode and vary the temporal delay between excitation and STED pulses till you achieve the lowest photon counts. Avoid multilamellar structures in SLB.

CRITICAL STEP: This step is not applicable for continuous wavelength (CW)-STED set-ups.

\section{Calibrating the diameters of the effective observation areas (Step 10):}

10. For imaging-based calibration using the fluorescent beads sample (for pSTED-FCS only), follow Option A. For FCS-based calibration using supported lipid bilayers, follow Option B (for pSTED-FCS) or Option C (for sSTED-FCS). See Experimental Design 'Calibration of the observation volume of the optical system' for detailed information on the different calibration strategies.

A. Calibration with beads

i. Take images of the beads sample with varying power of the STED laser $(0 \%$, $10 \%, 25 \%, 50 \%, 75 \%$ ).

ii. Select multiple isolated beads and measure FWHM with a line profile for example with ImageJ/FIJI. Get an average FWHM for each STED power and confocal case including at least 20 beads in your statistics.

iii. Plot FWHM vs. STED beam intensity.

CRITICAL STEP: Although FWHM values for different STED powers obtained using Option B is more reliable for later calculation of diffusion coefficients, for ideal STED dyes such as Atto $647 \mathrm{~N}$ or Abberior Star Red, the values obtained with Option A and Option B are comparable. Moreover, FWHM in confocal mode (STED power 0) should be obtained with this option using beads sample.

B. Calibration with supported lipid bilayer for pSTED-FCS

i. Place the SLB on the microscope and focus on a clear and homogeneous part of the SLB. Avoid multilamellar structures.

\section{TROUBLESHOOTING}

ii. $\quad$ Place your measurement cursor on the spot you would like to measure.

iii. Set the excitation laser power to $5 \mu \mathrm{W}$ for FCS measurements.

iv. First start with a confocal FCS measurement; acquire an autocorrelation curve without STED.

v. Switch on STED and repeat steps ii-iv using $10 \%$ of the total STED power 
CRITICAL STEP: The amplitude and the transit time of the correlation curve should shift when STED laser is on.

\section{TROUBLESHOOTING}

TROUBLESHOOTING

vi. Repeat step $\mathrm{v}$ for $20 \%, 50 \%$ and $75 \%$ STED power (percentages are only guidelines and can be chosen differently).

vii. Repeat the confocal measurement (step iv) on the same spot.

CRITICAL STEP: This step is crucial to test whether there is any change in membrane or focus during the acquisition. The first and the last measurements (two confocal measurements) should appear very similar (e.g. same amplitude and transit time). If there is a change, it is likely that the focus has changed during the acquisition which could be avoided with auto-focus control.

viii. Repeat steps ii-vii at 3 locations.

ix. Save all the data for analysis and calculation of the observation spot diameters (Step 13A). These FWHM values obtained using Eq.4 will be used later to calculate the diffusion coefficients in cell measurements at different STED powers using the Eq. 3 .

C. Calibration with supported lipid bilayer for sSTED-FCS

i. Prepare the SLB as described in Reagents Setup. In this case the SLB should be less fluid and contain about $50 \%(\mathrm{~mol} / \mathrm{mol})$ of cholesterol $(+50 \%$ of DOPC with a total lipid concentration of $1 \mathrm{mg} / \mathrm{mL}$ ).

ii. Place the SLB on the microscope and focus on a clear and homogeneous SLB. Avoid multilamellar structures.

iii. Select the area (line) to be measured.

iv. Set the excitation laser power to $10 \mu \mathrm{W}$ for $\mathrm{sFCS}$ measurements.

v. First start with a confocal sFCS measurement; acquire an intensity carpet without STED.

vi. Switch on STED and repeat step v using $10 \%$ of the total STED power

vii. Repeat step vi for $20 \%, 50 \%$ and $75 \%$ STED power (percentages are only guidelines and can be chosen differently).

viii. Repeat the confocal measurement (step v) on the same spot.

ix. Repeat steps iii-viii at 3 locations.

x. Save the images for later correlation (Step 13B) and calculation of effective observation spot sizes (Eq. 4), which will serve as the calibration for calculating diffusion coefficients from cell measurements at these STED powers using Eq. 3.

\section{TROUBLESHOOTING}

\section{Cell Labelling}

11. For labelling lipids, follow Option A, for labelling proteins, follow Option B.

\section{A. Lipid labelling (Timing 10 min)}


i. Wash the cells $2 \mathrm{x}$ with $1 \mathrm{~mL}$ L15 medium.

CRITICAL STEP: If your cells are sensitive, be gentle at this step to avoid detachment. Cells can also be washed with PBS or serum-free medium (depends on cell line and may require optimization).

ii. Mix $1 \mathrm{ml} \mathrm{L} 15$ medium with the fluorescent lipid analog of interest to a final concentration of $10-100 \mathrm{nM}$.

CRITICAL STEP: Use low volumes of lipids dissolved in ethanol. Avoid adding fluorescent lipid which is stored in methanol or DMSO as these solvent at higher concentration may cause damage to the cells.

CRITICAL STEP: High dye concentrations may affect the physical properties of membranes, thus these should always be kept to the minimal concentrations. Labelling always needs optimization with respect to the specific cell line and the fluorescent probe - vary timing, concentrations, medium/buffer conditions etc. Labelling can also be performed at $4{ }^{\circ} \mathrm{C}$ to further avoid internalization.

iii. Incubate the mixture for $10-20 \mathrm{~min}$ at room temperature $\left(21-25^{\circ} \mathrm{C}\right)$, or $5-10$ minutes at $37^{\circ} \mathrm{C}$.

iv. Wash the cells twice with $1 \mathrm{ml} \mathrm{L15}$ medium.

v. Add $1 \mathrm{~mL}$ of fresh $\mathrm{L} 15$ on the cells. For measurements at $37{ }^{\circ} \mathrm{C}$, use preheated L15.

vi. Perform imaging to check labelling.

CRITICAL STEP: Imaging and STED-FCS measurements (Step 12) should be done immediately after the labelling and measurement time should be kept short $(\approx 1 \mathrm{~h})$ to ensure that the cells are healthy.

TROUBLESHOOTING

TROUBLESHOOTING

\section{B. Protein Labelling (Timing 1 h)}

CRITICAL: The Procedure in this option is for labelling cells expressing Halo/SNAP-tagged proteins of interest. Please refer to Box 1 for optimal labelling strategies.

i. Wash the cells $2 \mathrm{x}$ with $1 \mathrm{~mL}$ L15 medium.

CRITICAL STEP: If your cells are sensitive, be gentle at this step to avoid detachment. Cells can also be washed with PBS or serum-free medium (depends on cell line and may require optimization).

ii. $\quad$ Mix $1 \mathrm{ml}$ of full media (growth medium supplemented with serum) with Halo/SNAP ligand and add the mixture into the dish. 
CRITICAL STEP: The concentration of the Halo/SNAP ligands should be optimized for each protein and each tag as it can vary significantly from the manufacturer's proposed concentration (usually $1 \mu \mathrm{M}$ ). According to our experience, $100 \mathrm{nM}$ of TMR-Halo for instance yields better signal to noise ratio. Higher concentration may increase the level of background and cause increased surface binding.

iii. Incubate the cells at $37^{\circ} \mathrm{C}$ for $30 \mathrm{~min}$.

iv. $\quad$ Rinse cells $2 \times$ with full media $(2 \times 30 \mathrm{~min})$.

v. Incubate cells with full media at $37^{\circ} \mathrm{C}$ for $30 \mathrm{~min}$.

vi. Rinse cells $2 \mathrm{x}$ with $\mathrm{L} 15$.

vii. Put $1 \mathrm{ml}$ of fresh $\mathrm{L} 15$ onto the cells.

viii. Perform imaging to check labelling

TROUBLESHOOTING

TROUBLESHOOTING

\section{STED-FCS measurement.}

12. For data acquisition with point STED-FCS follow Option A, for scanning STED-FCS follow Option B.

\section{A. Point STED-FCS - TIMING: 2 h}

CRITICAL STEP: This Option requires an instrument that can do point FCS; it should be equipped with a single photon counting detector and a correlator or TCSPC electronics.

i. $\quad$ Place the cells on the microscope

ii. Focus on the fluorescently labelled cells.

CRITICAL STEP: Choose relatively dim cells, bright cells may not perform well for FCS. Avoid very bright spots, e.g. vesicles.

iii. $\quad$ Fine focus on the plasma membrane by maximising the intensity by moving the focus up and down.

CRITICAL STEP: Make sure that membrane is exactly in the centre of your focal volume, otherwise, signal (intensity counts) will be low, amplitude will be reduced, and diffusion will be skewed towards longer time scales. When the membrane is exactly in the centre of the focal spot; amplitude is highest, diffusion is fastest and signal is highest.

iv. Place your measurement cursor on the spot you would like to measure.

v. Set the excitation laser power to $5 \mu \mathrm{W}$ for FCS measurements.

vi. Start with a confocal FCS measurement; acquire an autocorrelation curve without STED. 
vii. Switch on STED and repeat step vi using $10 \%$ of the total STED power

viii. Repeat step vii for $20 \%, 50 \%$ and $75 \%$ STED power. Use the same powers as before during the SLB calibration (Step10B).

ix. Save all the data for analysis.

CAUTION: Use lowest excitation laser power possible to avoid photobleaching, saturation, phototoxicity and oxidation.

\section{B. Scanning STED-FCS - TIMING: 2 h}

i. $\quad$ Place the cells on the microscope

ii. Focus on the fluorescently labelled cells

iii. Select a region that is representative of the cell

CRITICAL STEP: Choose relatively dim cells, bright cells may not perform well for FCS. Avoid very bright spots, e.g. vesicles.

iv. Zoom in to around $5 \mu \mathrm{m} \times 5 \mu \mathrm{m}$ area.

v. Switch to $x t$ mode (line scan mode)

vi. Fine focus on the plasma membrane by maximising the intensity while moving up and down.

CRITICAL STEP: The length of the scanned line, the pixel size and the scanning frequency depend on the experiment and are subject to optimization. For sSTED-FCS experiments it is advisable to start with an observation spot size of $\sim 100 \mathrm{~nm}$, a respective pixel-size of $50 \mathrm{~nm}$, a scanning frequency of at least $2000 \mathrm{~Hz}$ and a line length of at least $1.5 \mu \mathrm{m}$.

vii. Set the pixel size to $20-50 \mathrm{~nm}$ (depends on STED power and calibration)

CRITICAL STEP: Pixel size may change; however it should be half of the smallest expected FWHM to ensure Nyquist sampling conditions for all measurements. Some commercial instruments can automatically adjust the pixel size to optimal; please check this with the supplier.

viii. Set the duration of the measurement to 90 seconds.

CRITICAL STEP: Duration can be adjusted depending on the signal, the longer the duration, the more the signal. Additionally the slower the observed dynamics, the longer the acquisition time needs to be. Once set, save the sSTED-FCS acquisition parameter and keep them constant for subsequent measurements.

ix. Acquire an $x t$ image first with no STED power (confocal scanning FCS measurement).

x. Switch on STED and repeat step ix using $10 \%, 20 \%, 50 \%$, and $75 \%$ of the total STED power; use the same values as in calibration in Step 10C. For sSTED-FCS, fewer points can be measured (e.g., $0 \%$ and $75 \%$ ).

xi. Save the images for analysis.

\section{TROUBLESHOOTING}




\section{Data analysis.}

13. For data analysis for point STED-FCS follow Option A, for scanning STED-FCS follow Option B.

CRITICAL: Please refer to refs ${ }^{21,79}$ for details on pSTED-FCS and sSTED-FCS software respectively. Also, we encourage readers to check the user manuals for the software where it is explained in detail how to operate them. Visit DOI:10.5281/zenodo.1314184 for pSTED-FCS and DOI: $10.5281 /$ zenodo. 1292334 for sSTED-FCS.

\section{A. Point STED-FCS data analysis - TIMING: $1 \mathrm{~h}$}

CRITICAL: pSTED-FCS data is analysed using FoCuS-point software (see the example video tutorial at https://bit.ly/2Ddy86L and Supplementary Figures S1-S4). For a software manual, go to DOI:10.5281/zenodo.1314184)

i. $\quad$ Run the FoCuS-point software

ii. Load the data from Steps $10 \mathrm{~B}(\mathrm{ix})$ and $12 \mathrm{~A}(\mathrm{ix})$. If the data is raw TSCPC data, they need to be loaded from the "Load TSCPC" tab. If the data is already correlated (for instance .fcs file or .sin file), go directly to the fitting tab (Step 13A(iv)).

iii. TCSPC data need to be gated to remove confocal contributions and ensure a more accurate amplitude of the resulting STED-FCS curve. Gate the TCSPC curves from 0.5 -1 ns after the maximum of the histogram until the full decay of the curve. Recalculate the autocorrelation curves. Depending on file sizes and computer performance, this step can take several minutes to hours. Using a CW-STED laser different time-gating windows (closer or further away from the TCSPC peak) will result in different effective observation spot sizes. The correlated curves will be exported to the "Fit Function" tab.

CRITICAL STEP: Apply the same gating for all the data.

iv. In fitting, select the appropriate fitting model (2D for membranes, number of components, triplet etc.). For membrane measurements, a singlecomponent 2D-diffusion model with a triplet is typically used.

v. Fix offset (untick the vary box) to 0 (or 1 depending on the file type, baseline for .fcs files is 1 instead of 0 ). If the triplet time of the used dye is known, fix the triplet time, e.g. $5 \mu$ s for Abberior Star Red-labelled lipids in membranes. Triplet amplitude can vary depending on STED power. Floating the alpha value (for anomalous sub-diffusion) is only advisable if the probe is expected to undergo interactions. In first instance it should be fixed to 1 , then can be varied from 0.7 to 1.1 .

vi. Fit current curve to check the fit quality. If necessary, allow variation of the offset as well. 
CRITICAL STEP: If the offset is large (compared to the FCS amplitude), this might indicate strong photobleaching, or occurrence of bright events. If the dataset was acquired in a time- or photon-mode, export time traces and apply photobleaching correction in the FoCuS-scan software, or crop out unwanted events. If acquired with the correlator, discard such measurements.

vii. Fit all curves and check each for the fit quality.

viii. Export the fit parameters to a file, e.g. an Excel spreadsheet.

ix. Use the fitted transit times at different STED powers to calculate the observation spot diameters (Eq. 4) or the apparent diffusion coefficients (Eq. 3) for the calibration and cell data, respectively. For pSTED-FCS calibration, the confocal FWHM is ideally obtained from the images of the beads sample (Step 10A) and the FWHM for varying STED powers with SLBs (Step 10B)

CRITICAL STEP: Pre-screen the obtained fit parameter values, especially the anomaly factors (alpha). While these are typically lower for STED than confocal FCS, values outside the range $0.7-1.1$ indicate non-homogeneous diffusion and should be interpreted with great care.

\section{B. Scanning STED-FCS data analysis - TIMING: $1 \mathrm{~h}$}

CRITICAL: sSTED-FCS data analysis is done using FoCuS_scan software (see the example video tutorial at https://bit.ly/2OGK4Ag and Supplementary Figures S5-S7). For a software manual, go to DOI:10.5281/zenodo. 1292334

i. Launch the FoCuS scan software

ii. Hit the "open file" button to load one or multiple measurements from Steps $10 \mathrm{C}(\mathrm{x})$ and $12 \mathrm{~B}(\mathrm{xi})$. Depending on file type and availability of metainformation, scanning frequency as well as pixel dwell time may need to be provided. The intensity data will be correlated while loading.

iii. Inspect the measurements using the time trace and the correlation carpet. Measurements with obvious artefacts (see Experimental Design "Controls and artefacts") should be removed or if possible cropped appropriately excluding the area with corrupted data.

CRITICAL STEP: For all measurements the first seconds should be removed by cropping, as they usually contain a considerable fraction of immobile bleaching molecules. If possible treat all data in the same way.

iv. Correct the datasets for photobleaching using either an exponential fit or the local averaging approach (if possible treat all data similarly).

v. Transfer the curves to the "Fit Function" tab.

vi. Fit the correlation carpets similarly to the pSTED-FCS curves. (Steps 13B(iv-vii) above). Normalization may be different, offset can be slightly varied due to duration noise.

vii. Export the fitted curves for further analysis. 
viii. Use the fitted transit times at different STED powers to calculate the observation spot diameters (Eq. 2) or the apparent diffusion coefficients (Eq. 3) for the calibration and cell data, respectively. For sSTED-FCS calibration, the confocal FWHM is ideally obtained from imaging the beads sample (Step 10A) and the FWHM for varying STED powers with SLBs (Step 10C).

CRITICAL STEP: Pre-screen the obtained fit parameter values, especially the anomaly factors (alpha). While these are typically lower for STED than confocal FCS, values outside the range 0.7-1.1 indicate non-homogeneous diffusion and should be interpreted with great care.

\section{Timing}

Step 1-3, preparation of the cells: 1-2 days

Steps 4-5, preparation of samples for alignment and calibration: $30 \mathrm{~min}$

Step 6-10, alignment and calibration of the system: $1 \mathrm{~h}$

Step 11 Option A, lipid labeling: $10 \mathrm{~min}$

Step 11 Option B, protein labeling: $1 \mathrm{~h}$

Step 12 Option A, point STED-FCS measurement: $2 \mathrm{~h}$

Step 12 Option B, scanning STED-FCS measurement: $2 \mathrm{~h}$

Step 13 Option A, point STED-FCS data analysis: $1 \mathrm{~h}$

Step 13 Option B, scanning STED-FCS data analysis: $1 \mathrm{~h}$

\section{Troubleshooting}

Possible problems and solutions are listed in Table 3.

\section{Table 3: Troubleshooting Table}

\begin{tabular}{|l|l|l|l|}
\hline Step & Problem & Possible Reason & Solution \\
\hline 8 & $\begin{array}{l}\text { Confocal and STED images } \\
\text { are not aligned }\end{array}$ & $\begin{array}{l}\text { Improper optical } \\
\text { alignment }\end{array}$ & $\begin{array}{l}\text { In the case of custom-built } \\
\text { setup, adjust the dichroic }\end{array}$ \\
\hline
\end{tabular}




\begin{tabular}{|c|c|c|c|}
\hline & & & $\begin{array}{l}\text { STED mirror to properly } \\
\text { overlap excitation and } \\
\text { depletion beams. For } \\
\text { commercial system repeat the } \\
\text { alignment procedure. }\end{array}$ \\
\hline $10 . \mathrm{B}(\mathrm{i})$ & No SLB & $\begin{array}{l}\text { Low label density; dirty } \\
\text { glass slide; }\end{array}$ & $\begin{array}{l}\text { Increase dye concentration, } \\
\text { use freshly cleaned glass; } \\
\text { clean the glass with plasma } \\
\text { cleaner; as an alternative } \\
\text { vesicle fusion method can be } \\
\text { tried. }\end{array}$ \\
\hline $10 . \mathrm{B}(\mathrm{v})$ & No STED effect & $\begin{array}{l}\text { Lack of time } \\
\text { synchronization }\end{array}$ & $\begin{array}{l}\text { Delay timing between STED } \\
\text { laser and excitation beam as } \\
\text { well as detector gating may } \\
\text { need to be adjusted. }\end{array}$ \\
\hline $10 . \mathrm{B}(\mathrm{v})$ & Excessively noisy STED ACF & $\begin{array}{l}\text { Bad profile of STED } \\
\text { laser; excitation by } \\
\text { STED }\end{array}$ & $\begin{array}{l}\text { Check the doughnut profile of } \\
\text { the STED beam imaging gold } \\
\text { beads in a reflection mode, } \\
\text { correct optics if necessary } \\
\text { (e.g. align phase plate with the } \\
\text { beam; if needed, consult with } \\
\text { the vendor of your } \\
\text { microscope). Select another } \\
\text { dye (see Experimental } \\
\text { Design). }\end{array}$ \\
\hline 11.A(vi) & Too much internalisation & Fast endocytosis & $\begin{array}{l}\text { Optimise labelling protocol: } \\
\text { E.g. Increase dye } \\
\text { concentration and reduce } \\
\text { incubation time or label at } \\
\text { lower temperatures. }\end{array}$ \\
\hline 11.A(vi) & No labelling & $\begin{array}{l}\text { Suboptimal labelling } \\
\text { buffer }\end{array}$ & $\begin{array}{l}\text { Make sure there is no serum } \\
\text { in labelling medium; try } \\
\text { different labelling media; try } \\
\text { BSA coupling as suggested } \\
\text { here }^{19}\end{array}$ \\
\hline 11.B(viii) & No labelling & Non-ideal ligands & $\begin{array}{l}\text { Make sure you use cell } \\
\text { permeable Halo/SNAP ligand; } \\
\text { increase ligand concentration }\end{array}$ \\
\hline 11.B(viii) & Too much background & $\begin{array}{l}\text { Unbound ligands inside } \\
\text { the cell }\end{array}$ & $\begin{array}{l}\text { Repeat the washing step } \\
\text { multiple times more; decrease } \\
\text { the dye concentration }\end{array}$ \\
\hline 12.A(vi) & No signal & $\begin{array}{l}\text { Bad focusing; } \\
\text { insufficient number of } \\
\text { photons }\end{array}$ & $\begin{array}{l}\text { Make sure the laser is focused } \\
\text { on the membrane; increase the } \\
\text { laser power; choose another }\end{array}$ \\
\hline
\end{tabular}




\begin{tabular}{|l|l|l|l|}
\hline 12.A(vi) & Bleaching & $\begin{array}{l}\text { measurement location or a } \\
\text { different cell }\end{array}$ \\
\hline 12.B(xi) & $\begin{array}{l}\text { Decreasing intensity in the xt } \\
\text { carpet }\end{array}$ & Bleaching or focus drift & $\begin{array}{l}\text { Poory to use more photostable } \\
\text { dyes (see Experimental } \\
\text { Design); decrease the laser } \\
\text { power; if recorded in time- or } \\
\text { photon mode, apply } \\
\text { photobleaching correction in } \\
\text { post-processing }\end{array}$ \\
& & $\begin{array}{l}\text { To avoid bleaching try to use } \\
\text { more photostable dyes (see } \\
\text { Experimental Design); } \\
\text { decrease the laser power; } \\
\text { decrease the number of pixels. } \\
\text { Apply photobleaching } \\
\text { correction in post-processing. } \\
\text { To avoid focus drift during } \\
\text { acquisition, make sure } \\
\text { thermal equilibrium is reached } \\
\text { before starting the } \\
\text { measurement. }\end{array}$ \\
\hline
\end{tabular}

\section{Anticipated results}

As highlighted before, acquisition of robust STED-FCS data requires careful calibration of the instrument and thoughtfully chosen experimental conditions. Below, we demonstrate two examples of such conditions potentially yielding sub-optimal results: due to temporal misalignment between the STED and excitation lasers (Figure 4a-c), and due to slight defocusing (Figure 4d-f). In addition, we provide example results for both pSTED-FCS and sSTED-FCS and discuss how to troubleshoot the most common artefacts.

Alignment: Temporal misalignment - After having achieved a perfect spatial overlap of the STED and excitation beams (as shown in Figure 3a and described in Step 6-8) by imaging immobilised fluorescent beads, the temporal synchronisation of their pulsing has to be optimised either through imaging using the same beads (Figure 3c, Step 9A) ${ }^{90}$, or with FCS of freely diffusing dye, as described in Step 9B (Procedure). At the optimal delay setting with the most efficient depletion of the signal from the periphery of the confocal observation volume, the detected count-rates of the fluorescent molecules will be the lowest ( 0 ps is optimal; Figure 4a) while the autocorrelation 
function (ACF) amplitude will be the highest (Figure 4b) and transit time the shortest (Figure 4c). This ensures the best possible spatial resolution. It is advisable to run this optimisation with a well warmed-up setup to prevent any drifts, and to test your instrument for stability over time - at least for the length of the full experimental day. Dyes with very different lifetimes might also require slightly different settings. Note that STED-FCS results are more sensitive to slight temporal misalignment when using a STED laser with shorter pulse widths (examples shown below are for relatively long 700-ns pulses).

Alignment: Focus drift - As in confocal $\mathrm{FCS}^{53}$, measurements of molecular diffusion in 2D planar membranes require careful focusing of the observation volume on the sample. This is typically reliably achieved by maximising the intensity in the confocal mode, which then yields the highest count-rates in STED-FCS ( $0 \mathrm{~nm}$ is optimal position; Figure 4d), highest amplitudes of ACFs (Figure 4e) and shortest transit times (Figure 4f). Even though STED-FCS is slightly less sensitive to the defocusing than confocal $\mathrm{FCS}^{36}$, accurate focusing ensures less scattering of the results, which is especially valuable for cell experiments. Depending on the stability of the used setup, fine refocusing may be required before every measurement. Potential drifts of the focus during the measurement will especially affect sSTED-FCS experiments with longer acquisition times, for which an autofocusing device provided by some of the vendors of the microscopes may be helpful.

Diffusion laws in cells - Following the demonstration of the initial calibration of the STED-FCS experiment, we show below the results of exemplary pSTED-FCS and sSTED-FCS measurements in live PtK2 cells. We exemplify three different diffusion modes for three different molecules; fluorescently labelled phospholipids (DPPE) and sphingomyelin (SM) analogues, and GPIanchored proteins (GPI-APs) exhibiting free, trapping, and domain-like diffusion, respectively.

For robust measurements, good labelling is needed, which is evaluated by confocal imaging prior to any STED-FCS measurements. Figure 5a-c show images of the basal membranes of PtK2 cells labelled with Atto647N-DPPE, Atto647N-SM, and GPI-SNAP-Abberior Star Red. For reliable STED-FCS measurements, areas with homogeneous staining are typically chosen, e.g. avoiding bright spots of internalised vesicles.

Figure 5d-f demonstrates normalised confocal and STED-FCS curves for these three molecules, respectively, acquired in pSTED-FCS modality. For a freely diffusing molecule such as DPPE, the difference between transit times for confocal and STED-FCS is the largest $(\approx 15$ times for the 
example at the chosen STED power $200 \mathrm{~mW}$, Figure 5d), resulting in a constant $D$ for the varying observation spot size $d$ (Figure $5 \mathrm{~g}$ ). For transiently immobilised molecules such as SM, the difference between transit times for confocal and STED is significantly smaller compared to freely diffusing molecules ( $\approx 6$ times in our example, Figure $5 \mathrm{e}$ ), yielding a pronounced decrease of the $D$ at smaller $d$ (Figure 5h). Finally, GPI-APs show domain-like diffusion where the difference in transit times is larger than for trapped diffusion but smaller than for free diffusion $(\approx 10$ times, Figure 5f). Accordingly, D decreases with smaller $d$, yet it exhibits a kink towards the smaller observation spots (Figure 5i).

Similar information can be obtained also from sSTED-FCS measurements, which offer additional insight into spatial variability of diffusivity. Figure 6 shows representative correlation carpets for fluorescent DPPE, SM and GPI-SNAP diffusing in the basal membranes of Ptk2 cells. Besides extracting the diffusion law, which can be determined by fitting all the ACFs in each carpet as discussed in the pSTED-FCS case, the sSTED-FCS modality allows also to highlight spatial heterogeneity in diffusion rates and modes. Compared to more homogenous DPPE diffusion (Figure 6a, b), strong fluctuations in STED transit times of SM (Figure 6c, d) and GPI-AP (Figure $6 e, f)$ indicate their locally hindered diffusion.

Troubleshooting of the most common artefacts - The data presented above demonstrate typical STED-FCS measurements that provide meaningful and reliable information on molecular diffusion rates and modes. Not surprisingly, though, many datasets, especially those acquired in live cells, may suffer from various artefacts, which can bias the results and, if unspotted, may lead to erroneous conclusions. We, therefore, include below several examples of most commonly encountered problems, describe how to spot them, and advise on the means for their prevention, circumvention, or elimination.

The most common artefact in the STED-FCS measurements is photobleaching, which can be obvious from the decaying fluorescence intensity trace (arrow in Figure 7a). It causes a tail in the autocorrelation curves, which biases the correlation curves to longer time scales (Figure 7b, c). In order to avoid photobleaching, low laser powers and photostable dyes should be used (see Troubleshooting section). However, if unavoidable, the software for analysis of sSTED-FCS data offers a few ways to correct for photobleaching. In the usual case, immobile particles are bleached in the initial few seconds. This temporal section can be cropped out using the temporally crop 
function of the software (See Supplementary Figure S8 and software manual). After the initial photobleaching is dealt with, the remaining mild photobleaching can still be resolved by means of the implemented photobleaching correction algorithms. The software provides two ways to perform this ${ }^{21}$; local averaging and bleaching fit correction (see the software manual for details in Materials section). Once these operations are also performed, the resulting corrected time trace can be used for further analysis (compare Figure 7a-c with 7d-f).

Note that the photobleaching correction built in the FoCuS-scan software can also be used with pSTED-FCS data acquired in the photon mode: the (time-gated) intensity time traces can be exported from FoCuS-point (to .tiff) and imported into FoCuS-scan as single-pixel intensity datasets $(t$-scan). The data acquired in the correlator mode cannot be corrected; the operator should therefore identify gradually decreasing photon count-rates during acquisition, or recognise photobleaching artefacts in ACFs (Figure 7c), and discard such data right away.

Another major problem in STED-FCS measurements are high-intensity signal bursts due to bright clusters or vesicles moving in and out of the focal volume (Figure 8a), which, similar to photobleaching, bias or even dominate the correlation curves towards longer time scales (Figure $8 \mathrm{~b}$ ). This artefact is particularly unavoidable in sSTED-FCS since the data cannot be visualised real-time and the acquisition time needs to be rather long making the observation of events such as the passing of a vesicle more likely. Therefore, we developed strategies to deal with this disturbance post-acquisition. Temporal cropping similar to photobleaching correction can be applied if the signal bursts are sparse and large part of the acquisition can be recovered by removing a few seconds of the measurement. However, if there are multiple signal bursts along the course of the whole measurement and hence temporal cropping cannot recover any reliable signal, the FoCuS-scan software also allows to spatially crop the datasets to discard the measured pixels that are affected by the bright particles; the rest of the pixels can be used for further analysis. Figure $8 \mathrm{~b}$ shows a correlation carpet where part of the pixels do not suffer from the signal burst (Figure 8c), while the other part shows extensive signal bursts (Figure 8d). In this case, the part without the signal bursts (Figure 8c) can be cropped and used for further analysis (see Supplementary Figure S8 or the software manual for detailed instructions).

Similarly as for previously described photobleaching correction, time-cropping of burst-affected photon-mode pSTED-FCS data can be performed after having exported the intensity time traces 
from FoCuS-point software. Irregularly shaped ACFs resulting from the data acquired in the correlator mode should be discarded during acquisition or analysis.

\section{Conflict of Interest}

Authors have no conflict of interest.

\section{Author Contribution}

All authors took part in acquiring and analysing the data and writing the manuscript.

\section{Data Availability Statement}

The STED-FCS data is available upon request. The software used for data analysis is freely available (see Equipment).

\section{Acknowledgements}

We thank the Wolfson Imaging Centre Oxford and the Micron Advanced Bioimaging Unit (Wellcome Trust Strategic Award 091911) for providing microscope facility and financial support. We acknowledge funding by the Wolfson Foundation, the Medical Research Council (MRC, grant number MC_UU_12010/unit programmes G0902418 and MC_UU_12025), MRC/BBSRC/EPSRC (grant number MR/K01577X/1), the Wellcome Trust (grant ref 104924/14/Z/14), the Deutsche Forschungsgemeinschaft (Research unit 1905 "Structure and function of the peroxisomal translocon") and Oxford-internal funds (John Fell Fund and EPA Cephalosporin Fund). ES is funded by the Newton-Katip Celebi Institutional Links grant (352333122). IU thanks the support from European Commission by Marie Skłodowska-Curie individual fellowship (707348).

\section{References}


1 Stefan, W. H. et al. The 2015 super-resolution microscopy roadmap. Journal of Physics D: Applied Physics 48, 443001 (2015).

2 Sahl, S. J., Hell, S. W. \& Jakobs, S. Fluorescence nanoscopy in cell biology. Nature reviews. Molecular cell biology 18, 685-701 (2017).

3 Elson, E. L. Fluorescence correlation spectroscopy: past, present, future. Biophys J 101, 28552870 (2011).

4 Sezgin, E. Super-resolution optical microscopy for studying membrane structure and dynamics. Journal of physics. Condensed matter : an Institute of Physics journal 29, 273001 (2017).

5 Hell, S. W. \& Wichmann, J. Breaking the diffraction resolution limit by stimulated emission: stimulated-emission-depletion fluorescence microscopy. Optics Letters 19, 780-782 (1994).

$6 \mathrm{Klar}$, T. A. \& Hell, S. W. Subdiffraction resolution in far-field fluorescence microscopy. Optics Letters 24, 954-956 (1999).

$7 \quad$ Klar, T. A., Jakobs, S., Dyba, M., Egner, A. \& Hell, S. W. Fluorescence microscopy with diffraction resolution barrier broken by stimulated emission. Proceedings of the National Academy of Sciences of the United States of America 97, 8206-8210 (2000).

8 Magde, D., Elson, E. L. \& Webb, W. W. Fluorescence correlation spectroscopy. II. An experimental realization. Biopolymers 13, 29-61 (1974).

9 Kim, S. A., Heinze, K. G. \& Schwille, P. Fluorescence correlation spectroscopy in living cells. Nature methods 4, 963-973 (2007).

10 Wawrezinieck, L., Rigneault, H., Marguet, D. \& Lenne, P. F. Fluorescence correlation spectroscopy diffusion laws to probe the submicron cell membrane organization. Biophys $J$ 89, 4029-4042 (2005).

11 Lenne, P. F. et al. Dynamic molecular confinement in the plasma membrane by microdomains and the cytoskeleton meshwork. The EMBO journal 25, 3245-3256 (2006).

12 Mueller, V. et al. STED nanoscopy reveals molecular details of cholesterol- and cytoskeletonmodulated lipid interactions in living cells. Biophysical Journal 101, 1651-1660 (2011).

13 Schneider, F. et al. Diffusion of lipids and GPI-anchored proteins in actin-free plasma membrane vesicles measured by STED-FCS. Molecular Biology of the Cell 28, 1507-1518 (2017).

14 Fujiwara, T. K. et al. Confined diffusion of transmembrane proteins and lipids induced by the same actin meshwork lining the plasma membrane. Mol Biol Cell 27, 1101-1119 (2016). Kusumi, A., Ike, H., Nakada, C., Murase, K. \& Fujiwara, T. Single-molecule tracking of membrane molecules: plasma membrane compartmentalization and dynamic assembly of raft-philic signaling molecules. Semin. Immunol. 17, 3-21 (2005).

16 Kusumi, A. \& Suzuki, K. Toward understanding the dynamics of membrane-raft-based molecular interactions. Biochimica Et Biophysica Acta-Molecular Cell Research 1746, 234-251 (2005).

17 Andrade, D. M. et al. Cortical actin networks induce spatio-temporal confinement of phospholipids in the plasma membrane - a minimally invasive investigation by STED-FCS. Scientific reports 5, 11454 (2015).

18 Kastrup, L., Blom, H., Eggeling, C. \& Hell, S. W. Fluorescence fluctuation spectroscopy in subdiffraction focal volumes. Phys Rev Lett 94, 178104 (2005).

19 Eggeling, C. et al. Direct observation of the nanoscale dynamics of membrane lipids in a living cell. Nature 457, 1159-1162 (2009).

20 Clausen, M. P. et al. A straightforward approach for gated STED-FCS to investigate lipid membrane dynamics. Methods (San Diego, Calif.), 67-75 (2015).

21 Waithe, D. et al. Optimized processing and analysis of conventional confocal microscopy generated scanning FCS data. Methods (San Diego, Calif.) 140-141, 62-73 (2018). 

Confocal and Stimulated Emission Depletion Nanoscopy Imaging. Nano letters 18, 4233-4240 (2018).

23 Vicidomini, G. et al. STED-FLCS: An Advanced Tool to Reveal Spatiotemporal Heterogeneity of Molecular Membrane Dynamics. Nano letters 15, 5912-5918 (2015).

24 Honigmann, A. et al. Scanning STED-FCS reveals spatiotemporal heterogeneity of lipid interaction in the plasma membrane of living cells. Nature Communications 5, 5412-5412 (2014). Benda, A., Ma, Y. \& Gaus, K. Self-Calibrated Line-Scan STED-FCS to Quantify Lipid Dynamics in Model and Cell Membranes. Biophys J 108, 596-609 (2015).

26 Wang, R. et al. A straightforward STED-background corrected fitting model for unbiased STEDFCS analyses. Methods (San Diego, Calif.) 140-141, 212-222 (2018).

27 Lanzano, L. et al. Measurement of nanoscale three-dimensional diffusion in the interior of living cells by STED-FCS. Nat Commun 8, 65 (2017).

28 Koenig, M. et al. in SPIE BiOS. 9 (SPIE).

29 Sezgin, E., Levental, I., Mayor, S. \& Eggeling, C. The mystery of membrane organization: composition, regulation and physiological relevance of lipid rafts. Nature Reviews Molecular Cell Biology 18, 361-374 (2017).

30 Bianchini, P., Cardarelli, F., Di Luca, M., Diaspro, A. \& Bizzarri, R. Nanoscale protein diffusion by STED-based pair correlation analysis. PLoS One 9, e99619 (2014).

31 Hedde, P. N. et al. Stimulated emission depletion-based raster image correlation spectroscopy reveals biomolecular dynamics in live cells. Nat Commun 4, 2093 (2013).

32 Ringemann, C. et al. Exploring single-molecule dynamics with fluorescence nanoscopy. New J Phys 11, 103054 (2009).

33 Sozanski, K., Sisamakis, E., Zhang, X. \& Holyst, R. Quantitative fluorescence correlation spectroscopy in three-dimensional systems under stimulated emission depletion conditions. Optica 4, 982-988 (2017).

34 Gao, P. \& Nienhaus, G. U. Precise background subtraction in stimulated emission double depletion nanoscopy. Opt Lett 42, 831-834 (2017).

35 Chojnacki, J. et al. Envelope glycoprotein mobility on HIV-1 particles depends on the virus maturation state. Nat Commun 8, 545 (2017).

36 Urbancic, I. et al. Lipid Composition but Not Curvature Is the Determinant Factor for the Low Molecular Mobility Observed on the Membrane of Virus-Like Vesicles. Viruses 10 (2018).

37 Sezgin, E. et al. Partitioning, diffusion, and ligand binding of raft lipid analogs in model and cellular plasma membranes. Biochimica Et Biophysica Acta-Biomembranes 1818, 1777-1784 (2012).

38 Sarangi, N. K., Ayappa, K. G. \& Basu, J. K. Complex dynamics at the nanoscale in simple biomembranes. Scientific reports 7, 11173 (2017).

39 Sarangi, N. K., Roobala, C. \& Basu, J. K. Unraveling complex nanoscale lipid dynamics in simple model biomembranes: Insights from fluorescence correlation spectroscopy in super-resolution stimulated emission depletion mode. Methods (San Diego, Calif.) 140-141, 198-211 (2018).

40 Sarangi, N. K., P, II, Ayappa, K. G., Visweswariah, S. S. \& Basu, J. K. Super-resolution Stimulated Emission Depletion-Fluorescence Correlation Spectroscopy Reveals Nanoscale Membrane Reorganization Induced by Pore-Forming Proteins. Langmuir 32, 9649-9657 (2016).

41 Honigmann, A., Mueller, V., Hell, S. W. \& Eggeling, C. STED microscopy detects and quantifies liquid phase separation in lipid membranes using a new far-red emitting fluorescent phosphoglycerolipid analogue. Faraday Discussions 161, 77-89 (2013).

42 Steshenko, O. et al. Reorganization of Lipid Diffusion by Myelin Basic Protein as Revealed by STED Nanoscopy. Biophys J 110, 2441-2450 (2016). 
43 Guzman, C. et al. The Efficacy of Raf Kinase Recruitment to the GTPase H-ras Depends on H-ras Membrane Conformer-specific Nanoclustering*. Journal of Biological Chemistry 289, 9519-9533 (2014).

44 Chelladurai, R., Debnath, K., Jana, N. R. \& Basu, J. K. Nanoscale Heterogeneities Drive Enhanced Binding and Anomalous Diffusion of Nanoparticles in Model Biomembranes. Langmuir 34, 16911699 (2018).

45 Jee, A. Y., Dutta, S., Cho, Y. K., Tlusty, T. \& Granick, S. Enzyme leaps fuel antichemotaxis. Proc Natl Acad Sci U S A 115, 14-18 (2018).

46 Zhang, X., Sisamakis, E., Sozanski, K. \& Holyst, R. Nanoscopic Approach to Quantification of Equilibrium and Rate Constants of Complex Formation at Single-Molecule Level. The journal of physical chemistry letters 8, 5785-5791 (2017).

47 King, J. T., Yu, C., Wilson, W. L. \& Granick, S. Super-resolution study of polymer mobility fluctuations near c*. ACS Nano 8, 8802-8809 (2014).

48 Lagerholm, B. C., Andrade, D. M., Clausen, M. P. \& Eggeling, C. Convergence of lateral dynamic measurements in the plasma membrane of live cells from single particle tracking and STED-FCS. J Phys D Appl Phys 50, 063001 (2017).

49 Kusumi, A., Shirai, Y. M., Koyama-Honda, I., Suzuki, K. G. N. \& Fujiwara, T. K. Hierarchical organization of the plasma membrane: Investigations by single-molecule tracking vs. fluorescence correlation spectroscopy. Febs Letters 584, 1814-1823 (2010).

50 Reina, F. et al. Complementary studies of lipid membrane dynamics using iSCAT and superresolved fluorescence correlation spectroscopy. J Phys D Appl Phys 51, 235401 (2018).

51 Sezgin, E. et al. A comparative study on fluorescent cholesterol analogs as versatile cellular reporters. J Lipid Res 57, 299-309 (2016).

52 Humpolickova, J. et al. Probing diffusion laws within cellular membranes by Z-scan fluorescence correlation spectroscopy. Biophys J 91, L23-25 (2006).

53 Steinberger, T., Machan, R. \& Hof, M. Z-scan fluorescence correlation spectroscopy as a tool for diffusion measurements in planar lipid membranes. Methods in molecular biology (Clifton, N.J.) 1076, 617-634 (2014).

54 Veerapathiran, S. \& Wohland, T. The imaging FCS diffusion law in the presence of multiple diffusive modes. Methods (San Diego, Calif.) 140-141, 140-150 (2018).

55 Jin, W., Simsek, M. F. \& Pralle, A. Quantifying spatial and temporal variations of the cell membrane ultra-structure by bimFCS. Methods (San Diego, Calif.) 140-141, 151-160 (2018).

56 Krieger, J. W. et al. Imaging fluorescence (cross-) correlation spectroscopy in live cells and organisms. Nat Protoc 10, 1948-1974 (2015).

57 Di Rienzo, C., Gratton, E., Beltram, F. \& Cardarelli, F. Fast spatiotemporal correlation spectroscopy to determine protein lateral diffusion laws in live cell membranes. Proc Natl Acad Sci U S A 110, 12307-12312 (2013).

58 Di Rienzo, C., Gratton, E., Beltram, F. \& Cardarelli, F. Spatiotemporal Fluctuation Analysis: A Powerful Tool for the Future Nanoscopy of Molecular Processes. Biophys J 111, 679-685 (2016).

59 Digman, M. A. \& Gratton, E. Imaging barriers to diffusion by pair correlation functions. Biophys J 97, 665-673 (2009).

60 Moens, P. D., Digman, M. A. \& Gratton, E. Modes of diffusion of cholera toxin bound to GM1 on live cell membrane by image mean square displacement analysis. Biophys J 108, 1448-1458 (2015).

61 Malacrida, L., Hedde, P. N., Ranjit, S., Cardarelli, F. \& Gratton, E. Visualization of barriers and obstacles to molecular diffusion in live cells by spatial pair-cross-correlation in two dimensions. Biomedical optics express 9, 303-321 (2018). 
Wenger, J. et al. Diffusion analysis within single nanometric apertures reveals the ultrafine cell membrane organization. Biophys J 92, 913-919 (2007).

63 Leutenegger, M. et al. Confining the sampling volume for Fluorescence Correlation Spectroscopy using a sub-wavelength sized aperture. Optics express 14, 956-969 (2006).

64 Regmi, R. et al. Planar Optical Nanoantennas Resolve Cholesterol-Dependent Nanoscale Heterogeneities in the Plasma Membrane of Living Cells. Nano letters 17, 6295-6302 (2017). Clausen, M. P. et al. Pathways to optical STED microscopy. NanoBiolmaging 1, 1-12 (2014). Hotta, J. et al. Spectroscopic rationale for efficient stimulated-emission depletion microscopy fluorophores. J Am Chem Soc 132, 5021-5023 (2010).

67 Rankin, B. R. et al. Nanoscopy in a living multicellular organism expressing GFP. Biophys J 100, L63-65 (2011).

68 Butkevich, A. N. et al. Fluorescent Rhodamines and Fluorogenic Carbopyronines for SuperResolution STED Microscopy in Living Cells. Angewandte Chemie (International ed. in English) 55, 3290-3294 (2016).

69 Mobarak, E. et al. How to minimize dye-induced perturbations while studying biomembrane structure and dynamics: PEG linkers as a rational alternative. Biochim Biophys Acta (2018).

70 Hughes, L. D., Rawle, R. J. \& Boxer, S. G. Choose your label wisely: water-soluble fluorophores often interact with lipid bilayers. PLoS One 9, e87649 (2014).

71 Moneron, G. et al. Fast STED microscopy with continuous wave fiber lasers. Optics express 18, 1302-1309 (2010).

72 Hense, A. et al. Monomeric Garnet, a far-red fluorescent protein for live-cell STED imaging. Scientific reports 5, 18006 (2015).

73 Strack, R. L. et al. A rapidly maturing far-red derivative of DsRed-Express 2 for whole-cell labeling. Biochemistry 48, 8279-8281 (2009).

74 Morozova, K. S. et al. Far-Red Fluorescent Protein Excitable with Red Lasers for Flow Cytometry and Superresolution STED Nanoscopy. Biophysical Journal 99, L13-L15 (2010).

75 Giepmans, B. N., Adams, S. R., Ellisman, M. H. \& Tsien, R. Y. The fluorescent toolbox for assessing protein location and function. Science 312, 217-224 (2006).

76 Gendreizig, S., Kindermann, M. \& Johnsson, K. Induced protein dimerization in vivo through covalent labeling. J Am Chem Soc 125, 14970-14971 (2003).

77 Stagge, F., Mitronova, G. Y., Belov, V. N., Wurm, C. A. \& Jakobs, S. SNAP-, CLIP- and Halo-tag labelling of budding yeast cells. PLoS One 8, e78745 (2013).

78 Maraspini, R., Beutel, O. \& Honigmann, A. Circle scanning STED fluorescence correlation spectroscopy to quantify membrane dynamics and compartmentalization. Methods (San Diego, Calif.) 140-141, 188-197 (2018).

79 Waithe, D., Clausen, M. P., Sezgin, E. \& Eggeling, C. FoCuS-point: Software for STED Fluorescence Correlation and Time-Gated Single Photon Counting. Bioinformatics (Oxford, England) (2015).

80 Muller, P., Schwille, P. \& Weidemann, T. PyCorrFit-generic data evaluation for fluorescence correlation spectroscopy. Bioinformatics (Oxford, England) 30, 2532-2533 (2014).

81 Theer, P., Mongis, C. \& Knop, M. PSFj: know your fluorescence microscope. Nature methods 11, 981-982 (2014).

82 Moffitt, J. R., Osseforth, C. \& Michaelis, J. Time-gating improves the spatial resolution of STED microscopy. Optics express 19, 4242-4254 (2011).

83 Wahl, M., Gregor, I., Patting, M. \& Enderlein, J. Fast calculation of fluorescence correlation data with asynchronous time-correlated single-photon counting. Optics express 11, 3583-3591 (2003).

84 Rossow, M. J., Sasaki, J. M., Digman, M. A. \& Gratton, E. Raster image correlation spectroscopy in live cells. Nat Protoc 5, 1761-1774 (2010). 

of proteome dynamics in living cells. Nature biotechnology 33, 384-389 (2015).

86 Enderlein, J., Gregor, I., Patra, D. \& Fitter, J. Art and artefacts of fluorescence correlation spectroscopy. Current pharmaceutical biotechnology 5, 155-161 (2004).

87 Bacia, K. \& Schwille, P. Practical guidelines for dual-color fluorescence cross-correlation spectroscopy. Nat Protoc 2, 2842-2856 (2007).

88 Hiramoto-Yamaki, N. et al. Ultrafast Diffusion of a Fluorescent Cholesterol Analog in Compartmentalized Plasma Membranes. Traffic 15, 583-612 (2014).

89 Schindelin, J. et al. Fiji: an open-source platform for biological-image analysis. Nature methods $\mathbf{9}$, 676-682 (2012).

90 Galiani, S. et al. Strategies to maximize the performance of a STED microscope. Optics express 20, 7362-7374 (2012).

91 Keppler, A. et al. A general method for the covalent labeling of fusion proteins with small molecules in vivo. Nature biotechnology 21, 86-89 (2003).

92 Gautier, A. et al. An engineered protein tag for multiprotein labeling in living cells. Chem Biol 15, 128-136 (2008).

93 Los, G. V. et al. HaloTag: a novel protein labeling technology for cell imaging and protein analysis. ACS Chem Biol 3, 373-382 (2008).

\section{Figure legends}

Figure 1 | Principles of STED-FCS. a) STED microscopy relies on depletion of spontaneous fluorescence emission from excited molecules (blue) via stimulated emission induced by an additional laser featuring a local intensity zero in the centre (purple), thereby creating a subdiffraction-sized effective observation volume. b) FCS measures the average transit time of diffusing fluorescent molecules (green) through the observation volume of a microscope (blue) from recordings of the temporal fluorescence intensity fluctuations and subsequent calculation of the temporal autocorrelation function $(\mathrm{ACF})$ of the fluctuations in the detected fluorescence signal. ACF decays with transit time $\tau_{D}$ (the average molecular transit time through the observation spot) and amplitude $1 / \mathrm{N}$ (with $\mathrm{N}=$ average number of fluorescent molecules in the observation volume). c) Sketch of nanoscale plasma membrane heterogeneity which can be detected with STED-FCS but not with a large confocal observation volume. d) STED-FCS results in a series of diffusion coefficient measurements from FCS recordings that are obtained at various differently-sized effective observation spots. This makes it possible to distinguish between free (Brownian) (purple) and hindered diffusion modes such as transient trapping (green), transient domain incorporation (orange) or hop diffusion (blue) on the relevant sub-diffraction spatial scales $(<200 \mathrm{~nm}$ observation spot sizes). 
Figure 2 | Schematic overview of the protocol.

Figure 3 | Alignment and calibration of the system. a) Representative confocal and STED microscopy images of immobilised fluorescent beads (40 $\mathrm{nm}$ fluorescent crimson beads, Step 4). The confocal and STED laser beams are well aligned when these images are perfectly centred (Step 6-8). b) A representative intensity line profile over single isolated bead from the images in panel (a) (green: confocal, magenta: STED) confirming the optimized alignment. These profiles can be used to obtain the full width at half maximum values $(d)$. c) Representative confocal (left, green) and STED (right panels, magenta) images of fluorescent beads for different time delays between the excitation and STED laser pulses (Step 9A); perfect timing is at $0 \mathrm{~ns}$. d) Representative STED image of an Abberior Star Red labelled fluorescent lipid analogue diffusing in a supported lipid bilayer (SLB, Step 5) composed of 1,2-dioleoyl-sn-glycero-3-phosphocholine (DOPC) lipid on a glass coverslip (labelled lipid concentration is $0.05 \mathrm{~mol} \%$ ). e) Representative normalised FCS curves taken from the sample in panel (d), acquired at different powers of the STED laser (Step 10B). The decay and the apparent transit time shifts to shorter time scales with increasing STED laser power. f) Representative STED-FCS calibration data (Step 13A): Dependence of the observation spot size on the power of the STED laser as determined by FCS on SLB. Scale bars are $0.5 \mu \mathrm{m}$. Data is the mean value of 10 measurements, error bars are standard deviation.

Figure 4 | Artefacts in STED-FCS due to temporal misalignment of the excitation and STED laser (Time sync) and defocusing (Focus shift). We show exemplary pSTED-FCS data of Abberior Star Red labelled DPPE diffusing in DOPC supported lipid bilayers (SLB). At optimal temporal alignment (Step 9B) of the STED and excitation lasers (magenta), (a) intensity time traces show the lowest photon count-rates, (b) FCS curves the highest amplitude, and (c) fitted parameters the shortest transit times. Slight delays in both direction (negative (blue) or positive (green)) leads to suboptimal curves and wrong fit parameters. At the optimal focus position (magenta), (d) photon count-rates and (e) FCS amplitudes are the highest and (f) transit times the shortest. Slight defocusing (100 nm; green and $200 \mathrm{~nm}$; blue) leads to suboptimal curves and wrong 
fit parameters. Data points and error bars in panels (c) and (f) represent means and standard deviations of 5-10 repeated measurements per condition.

Figure 5 | Representative pSTED-FCS data for fluorescent phospholipids, sphingolipids and GPI-APs. a-c) Representative confocal images of PtK2 cells stained with a) Atto647N-DPPE, b) Atto647N-sphingomyelin and c) GPI-SNAP labelled with Abberior Star Red, respectively. d-f) Representative confocal (green) and STED-FCS (magenta) curves for d) Atto647N-DPPE, e) Atto647N-sphingomyelin and f) GPI-SNAP labelled with Abberior Star Red; STED power 200 $\mathrm{mW}$, corresponding to the observation spot size of $50 \mathrm{~nm}$. g-i) Dependence of $D$ to observation spot size $d$ for g) Atto647N-DPPE, h) Atto647N-sphingomyelin and i) GPI-SNAP labelled with Abberior Star Red. Values of $d$ were determined from the SLB calibration sample in Fig. 3e (Step 10B). Scale bars are $10 \mu \mathrm{m}$. Data for panels $\mathrm{g}, \mathrm{h}$, and $\mathrm{i}$ is the mean value of 10 measurements, error bars are standard deviation. It is partially adapted from ref $^{13}$.

Figure 6 | Representative sSTED-FCS data for fluorescent phospholipids, sphingolipids and GPI-APs. Correlation carpets and curves for fluorescently labelled a, b) Atto647N-DPPE c, d) Atto647N-sphingomyelin and e, f) GPI-SNAP (labelled with Abberior STAR Red SNAP ligand) in the basal membrane of Ptk2 cells, acquired in confocal (top row) and STED mode (bottom row, STED power $\approx 50 \mathrm{~mW}$ ); spatial resolution of $\approx 100 \mathrm{~nm}$. In STED carpets, DPPE shows almost homogeneous shift in transit time whereas spikes of inhomogeneity show up for SM and GPISNAP. Insets in panels (b), (d) and (f) are the confocal and STED curves combined in a single graph. It is partially adapted from $\operatorname{ref}^{22}$.

Figure 7 | Impact of photobleaching on sSTED-FCS and troubleshooting. Representative sSTED-FCS data showing photobleaching. a) Fluorescence intensity trace, b) correlation carpet and c) correlation curves for the Atto647N-labelled DPPE in PtK2 cell membrane before the photobleaching corrections. d) Fluorescence intensity trace, e) correlation carpet and f) correlation curves after bleaching correction. Arrows in panel (a) and (c) show the effect of photobleaching on intensity time traces and correlation curves. 
Figure 8 | Impact of bright signal bursts on sFCS and troubleshooting. Representative sFCS data showing bright signal bursts. a) Fluorescence intensity trace and b) resulting correlation carpet for all spatial pixels. Intensity traces from the pixels within the indicated green and magenta dashed boxes show c) absence of bursts in one part of the $x$-range of the scan while d) the bursts dominate the other part. The pixels shown in panel (c) can be used for further analysis after bleaching correction.

\section{Tables}

Table 1. A selection of STED-FCS compatible organic dyes*

\begin{tabular}{|c|c|c|}
\hline Green emission & Orange emission & Red emission \\
\hline Atto488 $\left(\right.$ ref $\left.^{20}\right)$ & $\operatorname{Atto532(\text {ref}^{37})}$ & Abberior Star Red $\left(\right.$ ref $\left.^{19}\right)$ \\
\hline Topfluor (ref ${ }^{51}$ ) & Atto565 (unpublished) & Atto647N (ref $\left.{ }^{19}\right)$ \\
\hline $\begin{array}{lll}\text { Abberior } & \text { Star } & 488 \\
\text { (unpublished) } & & \end{array}$ & Atto594 (unpublished) & SNAP Si-Rhodamine $\left(\right.$ ref $\left.^{43}\right)$ \\
\hline Abberior Star $512\left(\right.$ ref $\left.^{51}\right)$ & $\begin{array}{lll}\text { Abberior } & \text { Star } & 600 \\
\text { (unpublished) } & & \end{array}$ & Abberior Star 635P $\left(\right.$ ref $\left.^{35}\right)$ \\
\hline
\end{tabular}

* Note that this is only a selection of dyes that have worked well in STED-FCS experiments based on our experience.

Table 2. Different methods to perform STED-FCS measurements

\begin{tabular}{|c|c|c|c|}
\hline $\begin{array}{c}\text { Characteristics } \\
\text { Approach }\end{array}$ & Time & Correlator & Photon \\
\hline $\begin{array}{c}\text { Additional } \\
\text { hardware }\end{array}$ & none (beam scanner & signal splitter and & TCSPC electronics \\
for scanning FCS) & correlator & \\
\hline Spatial sampling & single point or line & single point & single point or line \\
\hline
\end{tabular}




\begin{tabular}{|c|c|c|c|}
\hline $\begin{array}{l}\text { Temporal } \\
\text { resolution }\end{array}$ & $\begin{array}{l}\text { high }(\mu \mathrm{s}) \text { for point- } \\
\text { FCS, low }(\mathrm{ms}) \text { for } \\
\text { scanning FCS }\end{array}$ & $\operatorname{high}(\mu \mathrm{s})$ & very high $(<\mu \mathrm{s})$ \\
\hline $\begin{array}{c}\text { Max photon count- } \\
\text { rate }\end{array}$ & $\sim 50 \mathrm{MHz}$ & $\sim 1 \mathrm{MHz}$ & $\sim 1-50 \mathrm{MHz}$ \\
\hline Gating & defined at acquisition & not possible & full flexibility \\
\hline $\begin{array}{c}\text { FCS preview } \\
\text { during acquisition }\end{array}$ & no & yes & yes \\
\hline Cropping possible & yes & no & yes \\
\hline Output data & $\begin{array}{l}\text { time trace as image }(t \\
\text { or } x t)\end{array}$ & FCS curve as data file & $\begin{array}{l}\text { table of time-tagged } \\
\text { time-resolved photon } \\
\text { arrivals, as binary file }\end{array}$ \\
\hline File size & intermediate $(\mathrm{MB})$ & small $(\sim 50 \mathrm{kB})$ & large (100 MB-1GB) \\
\hline $\begin{array}{c}\text { Speed of } \\
\text { correlation }\end{array}$ & slow & fast (on-fly) & slow \\
\hline $\begin{array}{c}\text { Commercially } \\
\text { integrated }\end{array}$ & $\begin{array}{l}\text { all STED microscopes } \\
\text { (with a beam scanner } \\
\text { for scanning FCS) }\end{array}$ & $\begin{array}{l}\text { can be integrated into } \\
\text { any platform allowing } \\
\text { direct access to the } \\
\text { signal from detectors }\end{array}$ & $\begin{array}{l}\text { can be integrated in } \\
\text { commercial and } \\
\text { custom-built platforms }\end{array}$ \\
\hline Additional cost & none & approx. 10k EUR & several 10k EUR \\
\hline
\end{tabular}

\section{BOXES}

\section{Box 1: Tips for best protein-labelling strategies}

As FCS relies on the photon budget, best measurements for STED-FCS will require photostable and bright fluorophores. It is crucial to notice that most but not all of the widespread STED dyes perform well with STED-FCS. For instance Oregon Green has been shown to be a suitable dye for STED imaging, however its bleaching behaviour makes it unsuitable for STED-FCS ${ }^{20}$. Best dyes tested so far for STED-FCS are Atto488, Abberior Star 488, Topfluor, Abberior Star 512 in green range, Atto532, Atto565, Atto594 and Abberior Star 600 in orange range, Abberior Star Red, Abberior Star 635P, Silicone Rodamine (SiR) and Atto647N in the far-red range (see Table 1). 
One conventional way to label proteins is via fluorescent proteins. These, however, have not been satisfying for STED-FCS measurements due to the photostability issue. Therefore, different strategies have been developed to couple proteins with organic photostable dyes. SNAP-, Halo-, CLIP-labeling ${ }^{91-93}$ are amongst these protein labelling strategies. In this case organic dyes that are linked to a small group $\left(\mathrm{O}^{6}\right.$-benzylguanine derivatives for the SNAP-tag, $\mathrm{O}^{2}$-benzylcytosine derivatives for the CLIP-tag, and primary alkylhalides for the Halo-tag) can easily be attached to the proteins that carry the SNAP, Halo or CLIP tag. For this type of labelling there are a few practical issues to consider. Firstly, if the structure of interest is intracellular, the dyes should be cell permeable, whereas if the structure is extracellular, it is better to use cell impermeable dyes. Second issue would be the background. Once the dye is inside the cells, a fraction of it will not bind the proteins. This fraction needs to be washed thoroughly by adding washing steps (Step 11B). The remaining background after the washing steps depends on the unspecific binding of the dye to the internal structures. Thus, every dye needs to be checked for this. To reduce background, the concentration of the dyes can also be reduced. Finally, the background signal can stem from unspecific binding of the dye (particularly sticky hydrophobic dyes) to the glass slide reducing the contrast. This fraction will bleach quickly in the first FCS acquisition, however one should be careful to avoid the artefact that the bleaching of immobile particles can cause. Although the manufacturers suggest $\approx 1 \mu \mathrm{M}$, lower concentrations (50-100 nM) can yield higher signal to noise ratio.

\section{- $\quad$ END OF BOX 1 -}

\section{Supplementary Information}

- Supplementary figures 1-8 